University of New Hampshire

University of New Hampshire Scholars' Repository

Physics Scholarship

Physics

$3-1-2000$

\title{
Energetic magnetosheath ions connected to the Earth's bow shock: Possible source of cusp energetic ions
}

S. W. Chang

J. D. Scudder

J. F. Fennell

R. Friedel

R. P. Lepping

See next page for additional authors

Follow this and additional works at: https://scholars.unh.edu/physics_facpub

Part of the Physics Commons

\section{Recommended Citation}

Chang, S.-W., J. D. Scudder, J. F. Fennell, R. Friedel, R. P. Lepping, C. T. Russell, K. J. Trattner, S. A. Fuselier, W. K. Peterson, and H. E. Spence (2000), Energetic magnetosheath ions connected to the Earth's bow shock: Possible source of cusp energetic ions, J. Geophys. Res., 105(A3), 5471-5488, doi:10.1029/ 1999JA900468.

This Article is brought to you for free and open access by the Physics at University of New Hampshire Scholars' Repository. It has been accepted for inclusion in Physics Scholarship by an authorized administrator of University of New Hampshire Scholars' Repository. For more information, please contact Scholarly.Communication@unh.edu. 


\section{Authors}

S. W. Chang, J. D. Scudder, J. F. Fennell, R. Friedel, R. P. Lepping, C. T. Russell, K. J. Trattner, S. A. Fuselier, W. K. Peterson, and Harlan E. Spence 


\title{
Energetic magnetosheath ions connected to the Earth's bow shock: Possible source of cusp energetic ions
}

\author{
S.-W. Chang, ${ }^{1}$ J. D. Scudder, ${ }^{1}$ J. F. Fennell, ${ }^{2}$ R. Friedel, ${ }^{3}$ \\ R. P. Lepping, ${ }^{4}$ C. T. Russell, ${ }^{5}$ K. J. Trattner, ${ }^{6}$ S. A. Fuselier, ${ }^{6}$ \\ W. K. Peterson, ${ }^{6}$ and H. E. Spence ${ }^{7}$
}

\begin{abstract}
Plasma and magnetic field data detected by NASA GGS/Polar and Wind during the May 4, 1998, storm event are analyzed to demonstrate for the first time a causal relation between the magnetosheath energetic ions and bow shock magnetic geometry. Intense magnetosheath energetic ions observed upstream from the cusp are from the quasi-parallel bow shock and show properties indicating that they are a possible source of cusp energetic ions.
\end{abstract}

\section{Introduction}

Energetic $\left(\sim 10^{1}-10^{2} \mathrm{keV}\right)$ ions are often observed in the magnetosheath. Two dominant sources are the magnetosphere [e.g., Hones et al., 1972; West and Buck, 1976; Sibeck et al., 1987; Fuselier et al., 1991; Kudela et al., 1992] and the solar wind energized at the bow shock [e.g., West and Buck, 1976; Asbridge et al., 1978; Crooker et al., 1981; Fuselier et al., 1991]. To understand the origins of energetic magnetosheath ions would greatly facilitate the determination of the mass, momentum, and energy transfer processes within the geospace.

The escape of energetic magnetospheric particles into the magnetosheath at times occurs on interconnected magnetosphere-magnetosheath field lines that result from magnetic reconnection at the magnetopause [e.g., Speiser et al., 1981; Scholer et al., 1981]. However, it mostly occurs on a continuous basis through a leakage process of finite gyroradius effects [e.g., Croley et al., 1986; Sibeck et al., 1987]. This mechanism predicts that energetic ions leak from the magnetosphere at postnoon local times and energetic electrons at prenoon local times owing to the difference in their drift paths in the magnetosphere. These ions stream along magne-

\footnotetext{
${ }^{1}$ Department of Physics and Astronomy, University of Iowa Iowa City.

${ }^{2}$ The Aerospace Corporation, Los Angeles, California.

${ }^{3}$ Los Alamos National Laboratory, Los Alamos, New Mexico.

${ }^{4}$ NASA Goddard Space Flight Center, Greenbelt. Maryland.

${ }^{5}$ Institute of Geophysics and Planetary Physics, University of California, Los Angeles.

${ }^{6}$ Lockheed Martin Advanced Technology Center, Palo Alto, California.

${ }^{7}$ Center for Space Physics, Boston University, Boston, Massachusetts.
}

Copyright 2000 by the American Geophysical Union.

Paper number 1999JA900468.

0148-0227/00/1999JA900468\$09.00 tosheath magnetic field lines. Depending on the magnetic geometry, they may escape into the upstream region of the bow shock [e.g., Sarris et al., 1976; Luhmann et al., 1984].

The upstream and downstream regions of the quasiparallel bow shock (the angle between the average upstream magnetic field and the shock normal, $\theta_{B n}$, less than $45^{\circ}$ ) are often populated with energetic particles and magnetic waves and turbulence [e.g., Paschmann et al., 1979; Greenstadt at el., 1980; Bonifazi and Moreno, 1981; Crooker et al., 1981; Ipavich et al., 1981; Greenstadt, 1985; Luhmann et al., 1986; Möbius et al., 1987; Gosling et al., 1989; Fuselier et al., 1995, and references therein]. Beginning with the earliest observations, it was realized that the connection of the interplanetary magnetic field (IMF) to the bow shock is a necessary condition for the presence of energetic particles on the interplanetary field line [e.g., Asbridge et al., 1968; Scudder et al., 1973; Lin et al., 1974]. The fact that the enhanced plasma and magnetic turbulence and their associated energetic ions are similar in both the upstream and downstream regions led to the suggestion of the same bow shock source region [e.g., West and Buck, 1976; Asbridge et al., 1978]. The occurrence rate of enhanced energetic ion events increases with decreasing $\theta_{B n}$ both upstream and downstream from the bow shock [e.g., Bonifazi and Moreno, 1981; Crooker et al., 1981; Mitchell and Roelof, 1983]. It is widely accepted that solar wind ions undergo first-order Fermi acceleration by scattering back and forth across the quasi-parallel bow shock in the turbulent regions upstream and downstream from the shock [Lee, 1982; Ellison, 1985]. In situ plasma and magnetic field observations have confirmed theoretical predictions of the coupling between the particles and waves [Möbius et al., 1987; Trattner et al., 1994]. Energy spectra of energetic ions from computer simulations are also in a very good agreement with observations both upstream and downstream from the quasi-parallel shock [Ellison et al., 1990]. Bursts of magnetospheric ions are also occasionally observed in the upstream region, but they 
have a much harder spectrum than those of bow shock accelerated solar wind ions [Möbius et al., 1986].

Ion composition measurements have played a critical role in determining the relative contribution of bow shock accelerated ions and leakage of magnetospheric ions to the magnetosheath energetic ions [e.g., Fuselier et al., 1991]. The angular distribution of bow shock accelerated ions immediately downstream from the quasiparallel bow shock is anisotropic with the maximum flux toward the magnetopause [e.g., Scholer et al., 1989; Ellison et al., 1990]. They contribute to the majority of energetic $\mathrm{H}^{+}(>65 \%)$ and almost all the energetic $\mathrm{He}^{+2}$ in the magnetosheath downstream from the quasi-parallel shock [Fuselier, 1994]. Leakage of magnetospheric ions plays a minor role in this region but accounts for nearly all the energetic protons in the plasma depletion layer [Fuselier, 1992], a thin layer adjacent to the magnetopause just outside the magnetosphere in the magnetosheath [Zwan and Wolf, 1976].

Recent analysis of cusp energetic particles (CEPs) suggest that they are from the bow shock accelerated ions [Chang et al., 1998]. If this hypothesis is true, one would expect the appearance of bow shock accelerated ions in the magnetosheath upstream from the cusp. In this paper, we present evidence of magnetosheath energetic ions upstream from the cusp accelerated at the quasi-parallel bow shock using plasma and magnetic field data observed by the NASA GGS/Polar and Wind spacecraft during a magnetic storm event on May 4, 1998. In contrast to previous statistical work [e.g., Crooker et al., 1981; Fuselier, 1994] a causal relation between the magnetosheath energetic ions and bow shock magnetic geometry is demonstrated for the first time. This storm provides a great opportunity to understand the source of magnetosheath energetic ions and to check the hypothesis of bow shock as a source of CEPs by supplying large-scale temporal variations in the energetic ion fluxes which should be correlated with upstream parameters at the source region with a proper time delay. Applying the above principle to the high time resolution data from Polar and Wind, we are able to determine not only the origin of the observed magnetosheath energetic ions but also the upstream parameters that control the intensity of these ions.

The rest of the paper is organized as followed. We present the instrumentation in section 2, Polar observations of plasma composition and data interval selection for cross-correlation analysis in section 3 , solar wind propagation time in section 4, magnetosheath energetic ions and their correlation with the IMF cone angle in section 5 , interpretation of the analysis results in section 6, and summary and conclusions in section 7 .

\section{Instrumentation}

Energetic ion composition measurements during this storm event were made by the Magnetospheric Ion Composition Sensor (MICS) of the Charge and Mass Magnetospheric Ion Composition Experiment (CAMMICE) onboard Polar. A similar detector was flown on the CRRES satellite [Wilken et al., 1992]. The MICS sensor sampled two-dimensional angular distributions and covered the energy range from 1 to $193.4 \mathrm{keV} / e$ for $\mathrm{H}^{+}$and $\mathrm{He}^{+2}, 1$ to $100.1 \mathrm{keV} / e$ for $\mathrm{O}^{>+2}, 41.1$ to 193.4 $\mathrm{keV} / e$ for $\mathrm{O}^{<+3}$, and 17.5 to $193.4 \mathrm{keV} / \mathrm{e}$ for $\mathrm{He}^{+}$(hereinafter energetic ion energy referring to energy per unit charge). The $\Delta E / E$ for each energy channel is $\sim 10 \%$. It took $\sim 3.3 \mathrm{~min}$ to complete a full energy sweep. The $\mathrm{H}^{+}$data were the total ion measurements assuming $\mathrm{H}^{+}$ response in the double coincidence rate (DCR) channel of the MICS detector. The detector viewed with a narrow aperture of $3^{\circ} \times 3^{\circ}$ perpendicular to the spacecraft spin axis which was mainly in the dawn-dusk direction during this event. Energetic electron observations were made with the Imaging Electron Sensor (IES) of the Comprehensive Energetic Particle and Pitch Angle Distribution (CEPPAD) onboard Polar [Blake et al., 1995]. The sensor sampled 3-D distributions covering the energy range from 12 to $400 \mathrm{keV}$. The lower-energy part of the ion spectrum was provided by the Hydra spectrometer [Scudder et al., 1995] which sampled 3-D distributions of electrons and ions (assuming $\mathrm{H}^{+}$) covering the energy range from $\sim 10 \mathrm{eV}$ to $19 \mathrm{keV}$. The electron measurements from Hydra as well as the magnetic field measurements from the Magnetic Field Experiment (MFE) magnetometer [Russell et al., 1995] were used to identify regions of plasma sheet, magnetopause boundary layer, magnetosheath, and solar wind along the Polar trajectory. Upstream solar wind and IMF data were acquired with the Magnetic Field Investigation (MFI) magnetometer [Lepping et al., 1995] and the Faraday cup of the Solar Wind Experiment (SWE) [Ogilvie et al., 1995] both onboard the Wind spacecraft.

\section{Overview of Polar Observations}

On May 4, 1998, 0500-1200 UT, the Polar spacecraft was moving from $-0.3^{\circ}$ to $74.1^{\circ}$ magnetic latitude and from 4.4 to $9.0 R_{E}$ geocentric distance near magnetic local noon. The Wind spacecraft was $\sim 214 R_{E}$ upstream from the Earth, just north of the Sun-Earth line. A very strong interplanetary shock arrived at Wind at $\sim 0230$ UT. Wind observed large variations in the solar wind density $\left(\sim 4-65 \mathrm{~cm}^{-3}\right)$ and IMF strength $(\sim 5$ $40 \mathrm{nT}$ ) and high solar wind bulk speed $\sim 800 \mathrm{~km} / \mathrm{s}$ for the next 9 hours. Because of the high solar wind dynamic pressure (as large as $65 \mathrm{nP}$ ), the magnetosphere was severely compressed and bow shock even reached the Polar altitude on several occasions. As a result of the rapid boundary motion due to the dynamics of the storm, Polar frequently crossed the magnetopause current layer. However, Polar was in the high-latitude dayside magnetosheath most of the time during the second half period $\sim 0840-1200 \mathrm{UT}$ of the storm event. (Note only relevant Polar data are presented in this section to address the plasma composition and magnetosheath interval selection for the cross-correlation analysis. For detailed analysis of Polar plasma and field observations for this event, see Russell et al. [1999] and J. R. Wygant et al. (manuscript in preparation, 1999). 
Plate 1 presents from top to bottom panels MFE magnetic field magnitude, energy-time spectrograms for CAMMICE/MICS $\mathrm{He}^{+}, \mathrm{O}^{<+3}, \mathrm{O}^{>+2}, \mathrm{He}^{+2}, \mathrm{DCR}-\mathrm{H}^{+}$, Hydra ions, CEPPAD/IES electrons, Hydra electrons, and plasma regions traversed by Polar from 0500 to $1200 \mathrm{UT}$. The total energy coverages for $\mathrm{H}^{+}$and electron are $\sim 0.02-200 \mathrm{keV}$ and $\sim 0.01-400 \mathrm{keV}$, respectively. Before $0525 \mathrm{UT}$, Polar was in the plasma sheet characterized by a large magnetic field $(>220 \mathrm{nT})$ dominated by the positive $B_{z}$ component, and relatively intense energetic $\mathrm{He}^{+}(>20 \mathrm{keV} / e), \mathrm{O}^{<+3}(>40$ $\mathrm{keV} / e), \mathrm{H}^{+}(>40 \mathrm{keV})$, and electrons $(>20 \mathrm{keV})$, but relatively weak lower-energy (below $40 \mathrm{keV} / e$ ) $\mathrm{O}^{>+2}$, $\mathrm{He}^{+2}$, and $\mathrm{H}^{+}$fluxes. Polar then moved into the lowlatitude boundary layer (LLBL) and observed similar features before it exited the magnetopause into the magnetosheath at $\sim 0541$ UT [Russell et al., 1999]. Although the plasma sheet and LLBL are both on closed magnetic field lines with both feet anchored to the Earth, electron pitch angle distributions there are somewhat different, having trapped populations in the former region and counterstreaming distributions in the latter.

By contrast, in the magnetosheath from $\sim 0541$ to 0610 UT Polar observed disturbed weak magnetic field, enhanced $\mathrm{O}^{>+2}, \mathrm{He}^{+2}, \mathrm{H}^{+}$and $1-\mathrm{keV}$ ions and $100-\mathrm{eV}$ electrons but weak $\mathrm{O}^{<+3}$ and energetic electron flux (both nearly close to the background values). Then Polar reentered the LLBL/plasma sheet and observed plasmas with characteristics similar to those measured before 0541 UT. During the early part of the storm event from 0500 to 0730 UT, Polar was moving back and forth between the magnetosheath and the plasma sheet/LLBL across the magnetopause. After $0730 \mathrm{UT}$, Polar was in the magnetosheath and encountered the bow shock for the first time at $\sim 0735$ UT. Owing to the arrival of a brief, very strong pressure pulse (solar wind dynamic pressure $\sim 65 \mathrm{nP}$ ) the bow shock moved to $7.3 R_{E}$ at $38^{\circ}$ magnetic latitude and 1100 magnetic local time. Polar briefly stayed upstream from the shock and observed very cold solar wind ions and electrons as shown in the Hydra ion and electron spectrograms in Plate 1. It encountered the bow shock several times after that.

The bow shock crossings are more obviously illustrated in Figure 1, which shows the magnitude of the magnetic field from MFE and electron density from $\mathrm{Hy}$ dra covering 0730-1000 UT. Bow shock crossings are identified by the simultaneous, abrupt jumps in the two quantities. In addition, plasma bulk speed and temperature jumps also occurred corresponding to the magnetic field and electron density jumps. As Polar moved from the magnetosheath across the strong shock into the upstream region, a sharp increase in the bulk speed and a sharp decrease in the magnetic field, temperature, and density occurred [e.g., Russell et al., 1999]. Several upstream regions from the bow shock are identified and marked as the shaded regions in Figure 1. As the solar wind dynamic pressure quickly reduced, the bow shock and magnetopause retreated toward their normal positions and Polar crossed the magnetopause into the magnetopause boundary layer at $\sim 0755$ UT. Electron temperature then increased as expected.

The best signature, however, for distinguishing the boundary layer and magnetosheath region is the electron distribution since two regions have distinct magnetic topology, one for a closed magnetic field line and the other for a magnetosheath field line which is connected to the IMF and may or may not be connected to a geomagnetic field line. As an example shown in Figure $2 a$, electrons commonly show a counterstreaming distribution in the boundary layer, indicating a closed magnetic topology. Another very useful diagnostic of the magnetopause current layer as a rotational discontinuity is the generalized Walén test [Scudder et al., 1999a]. Magnetopause crossings identified using this technique are consistent with the findings based on the electron distributions [Scudder et al., 1999b]. After 0755 UT, Polar frequently crossed the magnetopause before exiting the boundary layer at 0836 UT. After that, except several brief upstream regions from the bow shock, Polar was in the magnetosheath until the end of the magnetic storm at $\sim 1200$ UT. The common electron distribution in the magnetosheath is nearly isotropic, accompanied by a weak field-aligned beam at low energy as shown in Figure 2b.

As shown in Plate 1, energetic ions especially $\mathrm{O}^{<+3}$ show distinct relative intensities in the magnetosheath and plasma sheet/LLBL. These energetic $\mathrm{H}^{+}, \mathrm{He}^{+2}$, and $\mathrm{O}^{>+2}$ magnetosheath ions are likely of the solar wind origin. During this storm event, most of the magnetosheath energetic ion fluxes show significant variations (see, for example, $50 \mathrm{keV} / e$ ions in the fourth to the sixth panel of Plate 1). However, the energetic $\mathrm{He}^{+}$ fluxes are low and remain relatively steady. Energetic ion fluxes observed upstream in the solar wind by the 3D Plasma instrument [Lin et al., 1995] onboard Wind were quite uniform. In addition, these fluxes are $\sim 1$ to 3 orders of magnitude lower than the magnetosheath energetic ion fluxes at the same energies observed by Polar. Therefore the solar energetic particles were not a direct source of the Polar observations. To understand the cause of the variations and ultimately the origin of these magnetosheath energetic ions, we choose the later, long period of magnetosheath traversal (0840-1200 UT excluding upstream intervals) for systematic analysis.

\section{Solar Wind Propagation Time}

If the source of the magnetosheath energetic ions is at a distance, the timing for the transport is a crucial factor. Since their composition suggests a solar wind source, solar wind propagation time becomes the next obvious quantity to examine. The propagation time was estimated by four different methods utilizing the plasma and field measurements from Wind and Polar. The first simple estimate used the measured solar wind bulk speed from Wind/SWE which was quite uniform $\sim 770 \mathrm{~km} / \mathrm{s}$ from 0650 to $0730 \mathrm{UT}$. An estimated propa- 


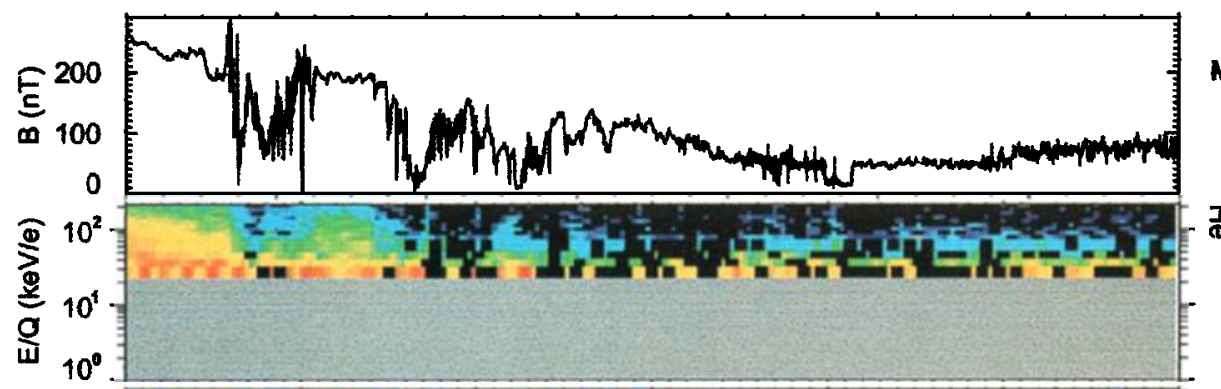

MFE B Field
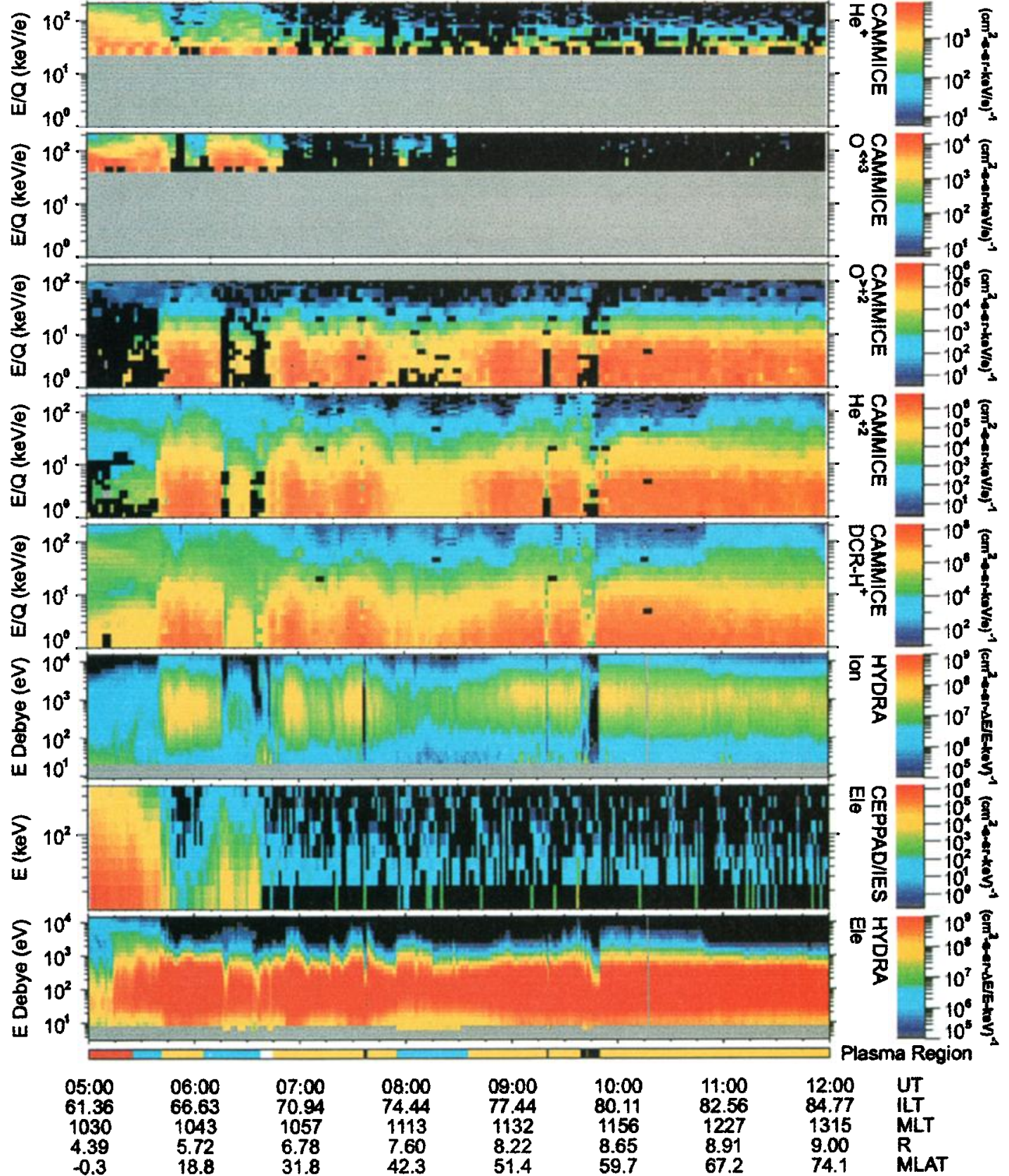

Plate 1. Magnetic field and plasma measurements from four instruments onboard Polar from 0500 to 1200 UT on May 4, 1998: (top to bottom) MFE magnetic field magnitude, energytime spectrograms for CAMMICE/MICS $\mathrm{He}^{+}, \mathrm{O}^{<+3}, \mathrm{O}^{>+2}, \mathrm{He}^{+2}, \mathrm{DCR}-\mathrm{H}^{+}$, Hydra ions, CEPPAD/IES electrons, Hydra electrons, and plasma regions traversed by Polar. Owing to the dynamic nature of this event, various regions such as plasma sheet (red), boundary layer (blue), magnetosheath (yellow), and upstream region of bow shock (black) were observed as indicated in the lower trace. 


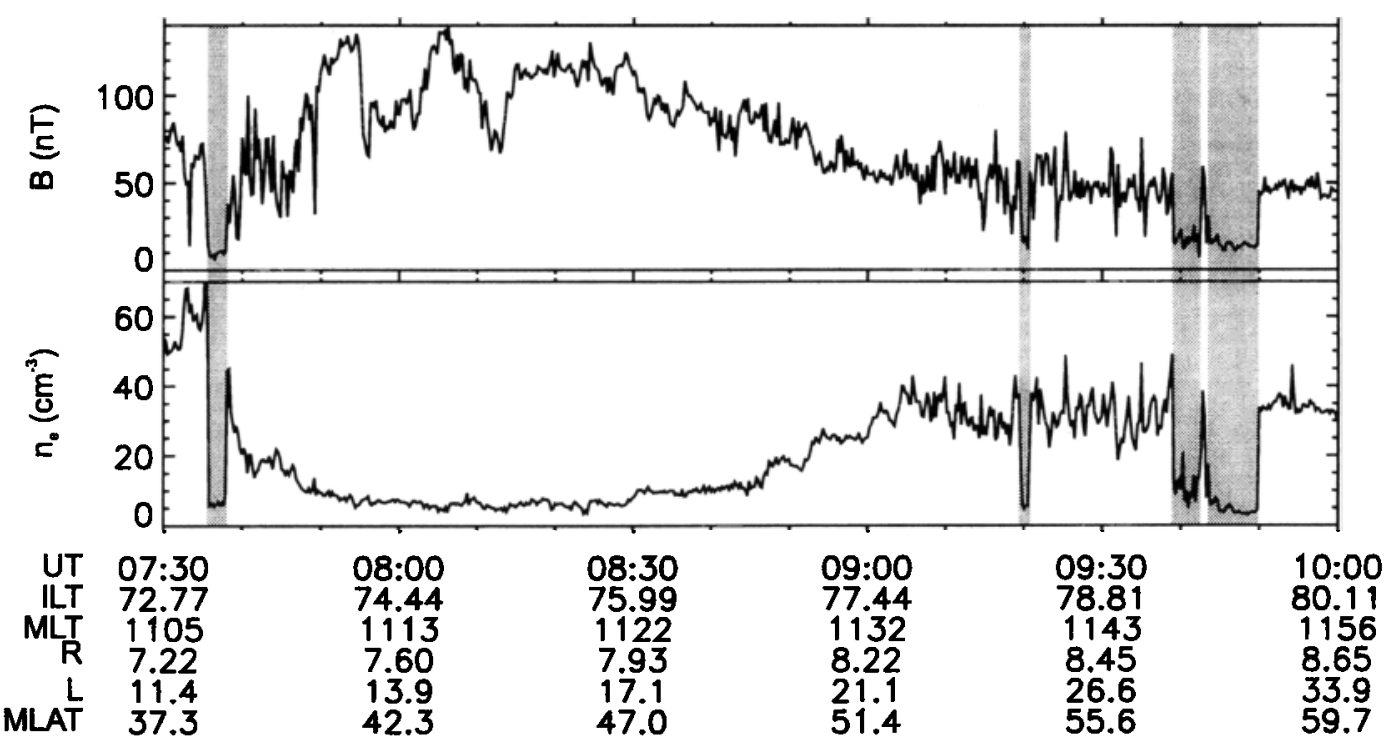

Figure 1. Polar/MFE magnetic field magnitude and Hydra electron density from 0730 to 1000 UT. Polar was mainly in the dayside magnetosheath and briefly crossed the bow shock into the upstream region (shaded) several times as indicated by the simultaneous, abrupt jumps in the two quantities.

gation time of $28 \mathrm{~min}$ was found for solar wind plasmas traveling a distance of $204 R_{E}$ from Wind to bow shock.

The Polar magnetic field profile observed in the upstream region of the bow shock from 0735:47 to 0738:03 $\mathrm{UT}$ is unique to the Wind IMF data set so that a comparison of IMF measurements from two spacecraft can give a fairly accurate estimate of the solar wind propagation time. Figure 3 presents the magnetic field measurements of Polar/MFE and Wind/MFI after an offset 27.3 min has been applied. The scales of all the vertical axes are set to facilitate the comparison and therefore most of the Polar measurements are off scale. In the upstream region, $B_{x}$ and $B_{z}$ components from Polar both turned from positive to negative at about the same time. An identical feature was also observed by Wind $\sim 27.3 \mathrm{~min}$ ahead. All the three components and magnitude of IMF from Polar and Wind (shaded region) match very well, with the exception of the $B_{y}$ components which show different trends but maintain the same sign. Within 2 hours ahead of this Polar upstream interval, only one match for all three IMF components occurs in the Wind data set. Other intervals have at best a match in one component only. The solar wind propagation time from Wind to Polar just upstream from the bow shock is thus 27.3 min consistent with the estimate from the solar wind bulk speed. For the time interval of interest, 0810-1130 UT, corresponding to the interval of the Polar magnetosheath energetic ion observations, the solar wind bulk speed decreased to a steady value $\sim 755 \mathrm{~km} / \mathrm{s}$ which gives an estimate of the propagation time of $\sim 29 \mathrm{~min}$ from Wind to bow shock. Additional several minutes are required for shocked solar wind to propagate from the shock to Polar in the magnetosheath because bow shock decelerates the flow.

Since magnetosheath magnetic field lines are connected to the IMF, magnetosheath field and IMF direc- tions are correlated despite magnetic field lines being deflected by the electric current at bow shock. Figure 4 presents the three magnetic field components observed by Polar from 0840 to 1200 UT and by Wind with a different lag indicated in each panel. These lags were determined by a cross-correlation analysis of the Polar and Wind observations during the above interval excluding the upstream regions from bow shock (shaded regions in Figure 4). Correlation coefficients for each magnetic field component are calculated for lags ranging from 0 to $60 \mathrm{~min}$ with an increment of $1 \mathrm{~min}$. All three correlation coefficient curves show one single peak with the correlation coefficients $(0.94,0.46,0.73)$ and time lags $\sim(35,34,37) \mathrm{min}$ for $\left(B_{x}, B_{y}, B_{z}\right)$ components. Strong correlation appears in $B_{x}$, a good correlation in $B_{z}$ but a weak correlation in $B_{y}$, as illustrated in Figure 4. From these results the estimated plasma propagation time from Wind to Polar is $~ 35-37 \mathrm{~min}$, which is consistent with the estimate using the solar wind bulk speed and magnetosheath plasma flow speed.

Another estimate of the propagation time is given by the cross-correlation analysis of the solar wind density and magnetosheath plasma density. Figure 5 presents electron density from Polar/Hydra from 0840 to 1200 UT and solar wind proton density from Wind/SWE offset $33 \mathrm{~min}$. The upstream regions (shaded) are again excluded in the analysis. As expected, magnetosheath plasma density (assuming charge neutrality) is strongly correlated with the solar wind density with a proper time delay. The correlation coefficient as a function of the lag from 0 to $60 \mathrm{~min}$ peaks at 0.85 for a lag of $33 \mathrm{~min}$, yielding another estimate of the solar wind propagation time. This peak, however, is not as sharp as those in the analysis of the magnetosheath magnetic field and IMF $B_{z}$ and $B_{x}$ components presented above. Therefore there is a somewhat larger uncertainty $( \pm 3 \mathrm{~min})$ in 

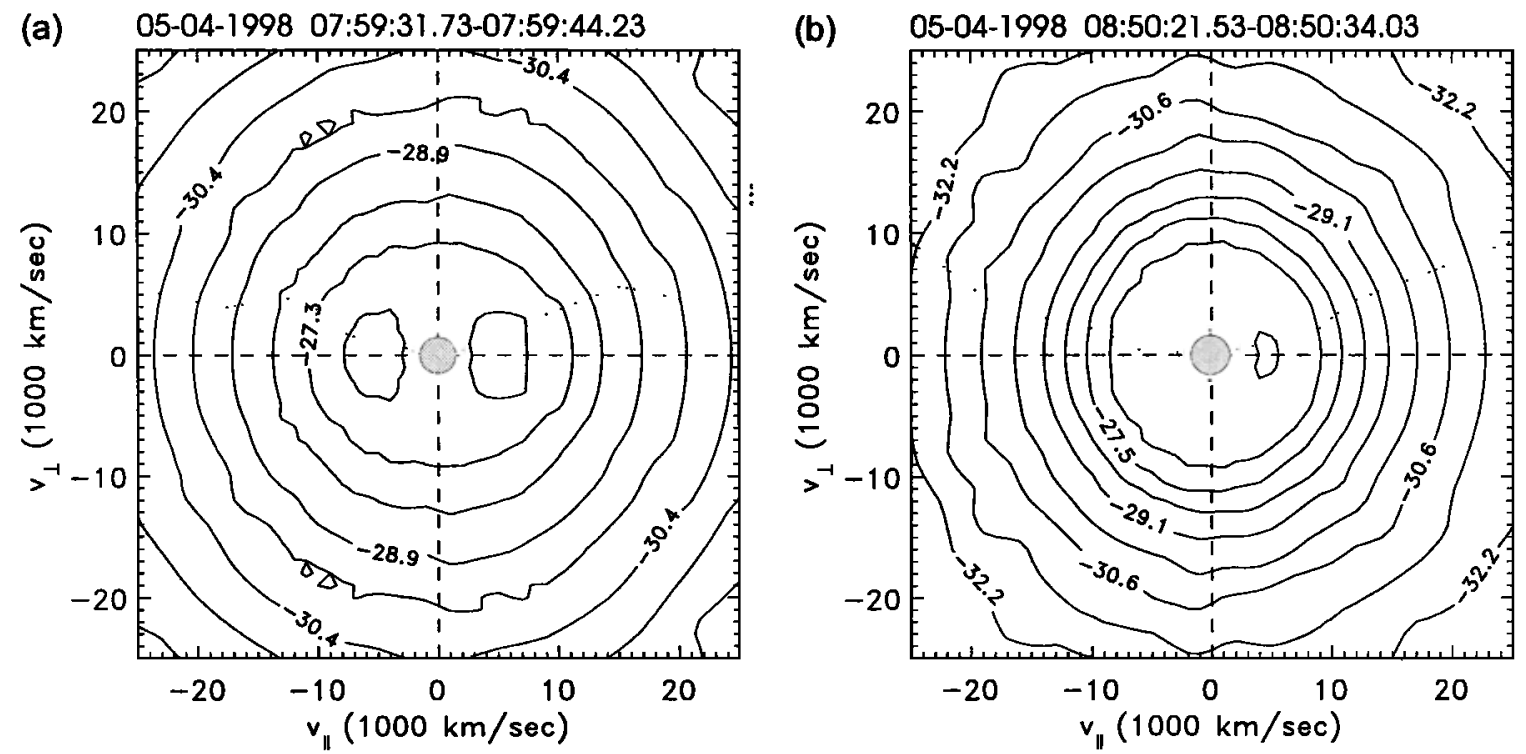

Figure 2. Contours of constant phase space density for electrons from Polar/Hydra in the plane of velocity components perpendicular and parallel to the magnetic field, showing (a) a counterstreaming distribution at energies below $\sim 500 \mathrm{eV}$ in the magnetopause boundary layer and (b) a nearly isotropic distribution with a weak field-aligned beam at $\sim 60 \mathrm{eV}$ in the magnetosheath. Contours are shown in the power of ten.

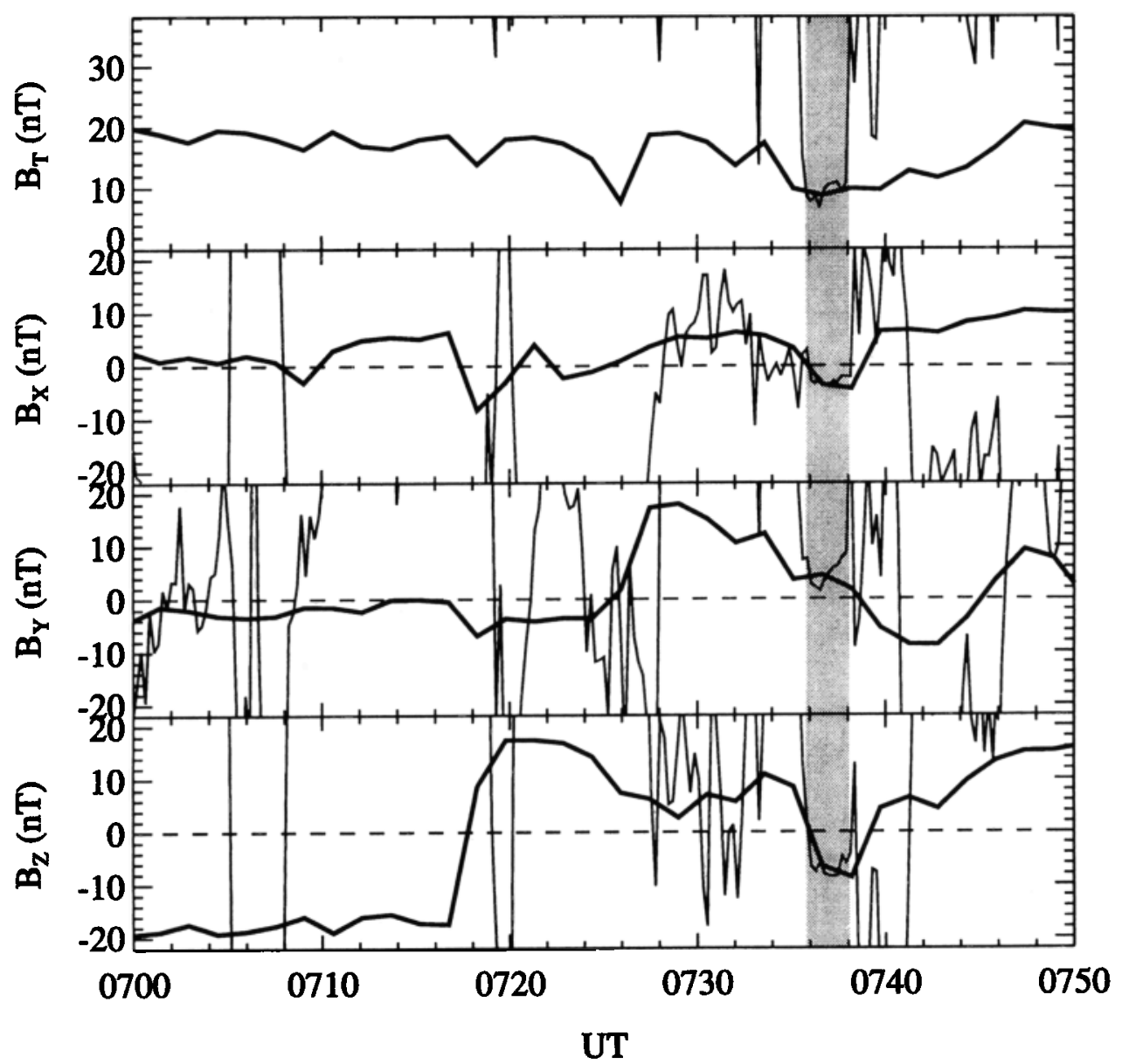

Figure 3. Magnitude and three GSM components of magnetic field observed by Polar/MFE (thin) from 0700 to $0750 \mathrm{UT}$ and Wind/MFI (thick) in the solar wind offset $27.3 \mathrm{~min}$. The IMF profile from Polar just upstream from the bow shock (shaded interval) matches the Wind observations very well. The scales of the vertical axes are set for an easy comparison of IMF profiles. Therefore MFE measurements are off scale most of the time. 


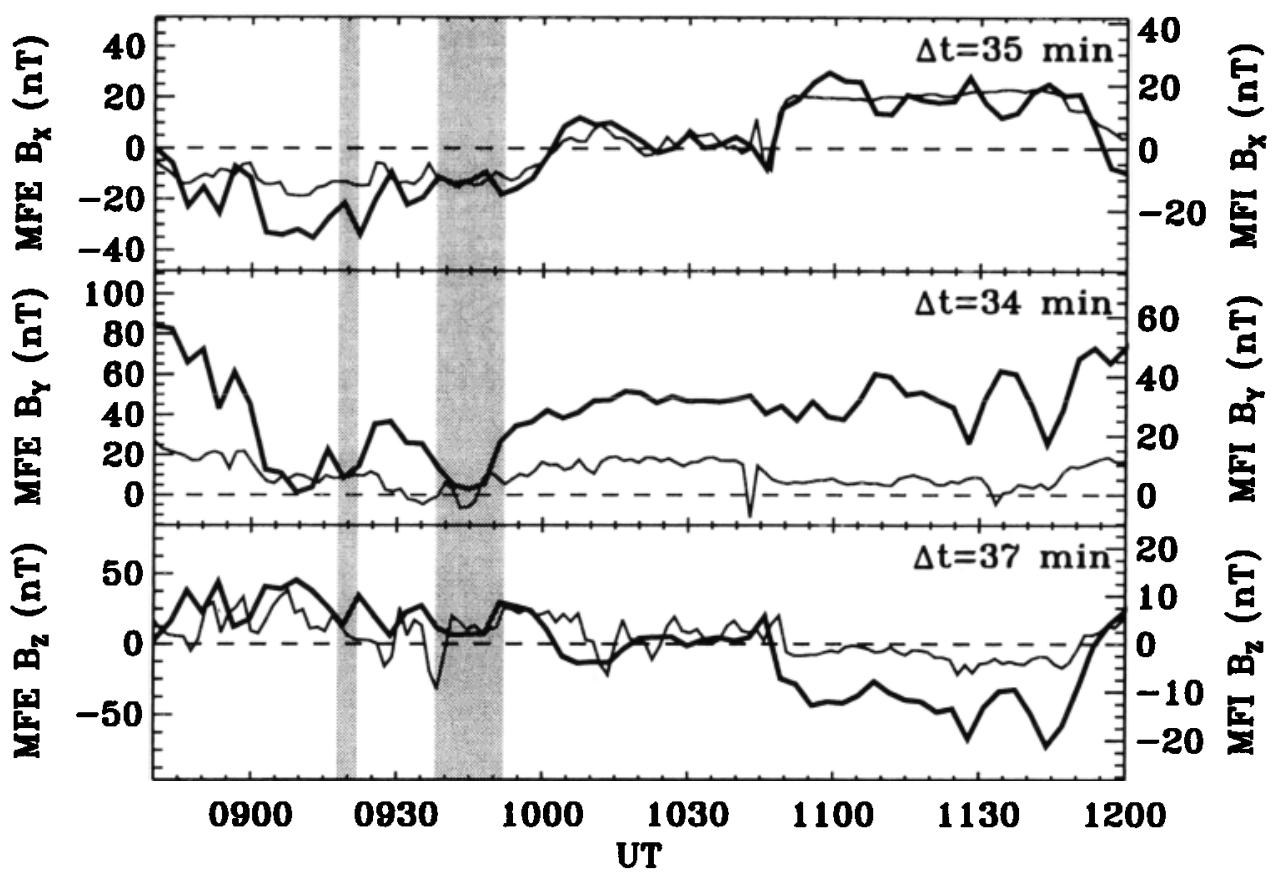

Figure 4. GSM magnetic field components in the magnetosheath from Polar/MFE (thick) from 0840 to 1200 UT and IMF components from Wind/MFI (thin) offset by $\Delta t$, which is the time delay associated with the peak correlation between the magnetosheath magnetic field and IMF. Data in the shaded areas are excluded in the cross-correlation analysis. Very strong correlation appears in $B_{x}$, a good correlation in $B_{z}$ but a weak correlation in $B_{y}$. Estimated solar wind propagation time is $\sim 35-37 \mathrm{~min}$.

this estimate. Nevertheless, the estimated propagation time is consistent with the one based on the magnetic field comparison.

\section{Magnetosheath Energetic Ions}

Figure 6 depicts energy spectra of $\mathrm{H}^{+}, \mathrm{He}^{+2}, \mathrm{O}^{>+2}$, and $\mathrm{He}^{+}$from Hydra and CAMMICE in the magnetosheath, averaged over an interval from $\sim 0840$ to $\sim 1200$ UT excluding the solar wind intervals. Ion fluxes are plotted against the ion energy per charge. Generally speaking, the two $\mathrm{H}^{+}$spectra agree well. Some differences may persist from different pitch angle averaging and detector efficiency for different ion species

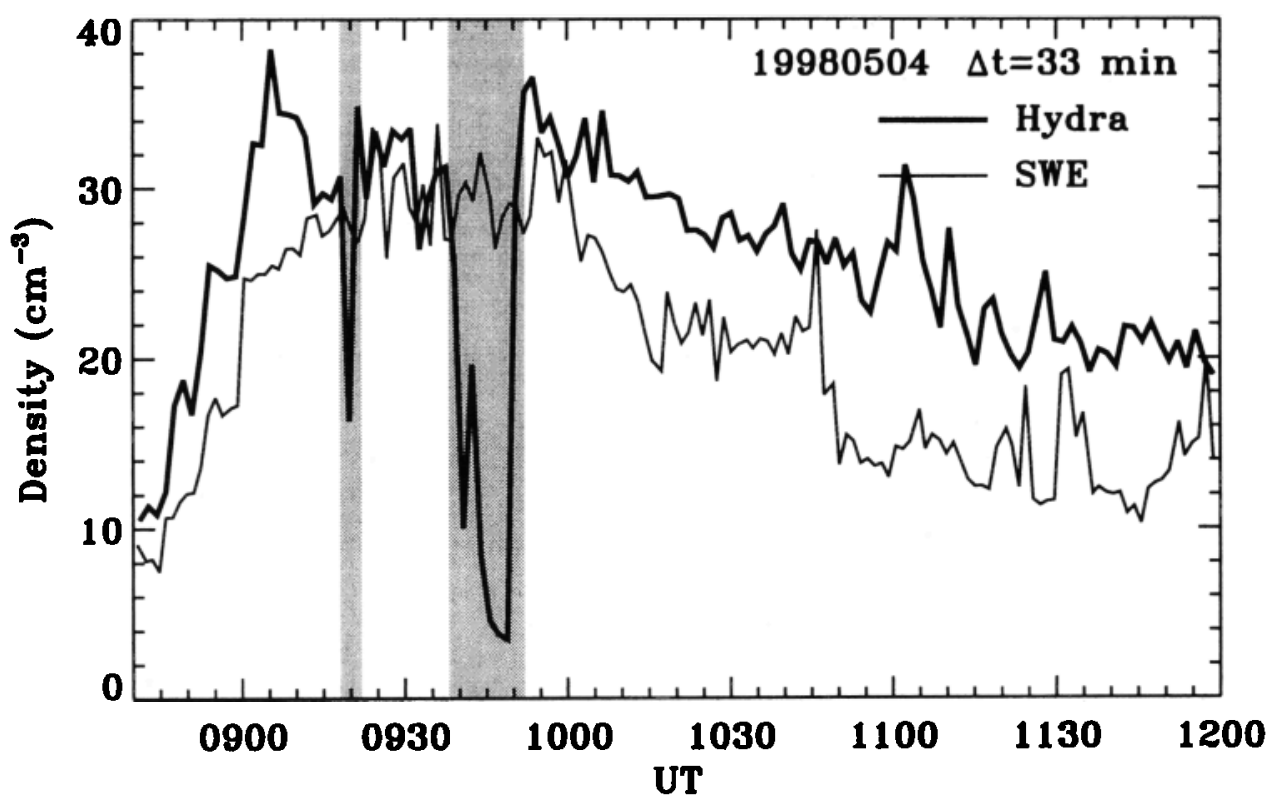

Figure 5. Polar/Hydra electron density in the magnetosheath (thick) from 0840 to $1200 \mathrm{UT}$ and the solar wind proton density from Wind/SWE (thin) offset $33 \mathrm{~min}$. Two quantities are strongly correlated. The 33-min lag associated with the best correlation gives an estimate of the solar wind propagation time. Data in the shaded areas are excluded in the cross-correlation analysis. 


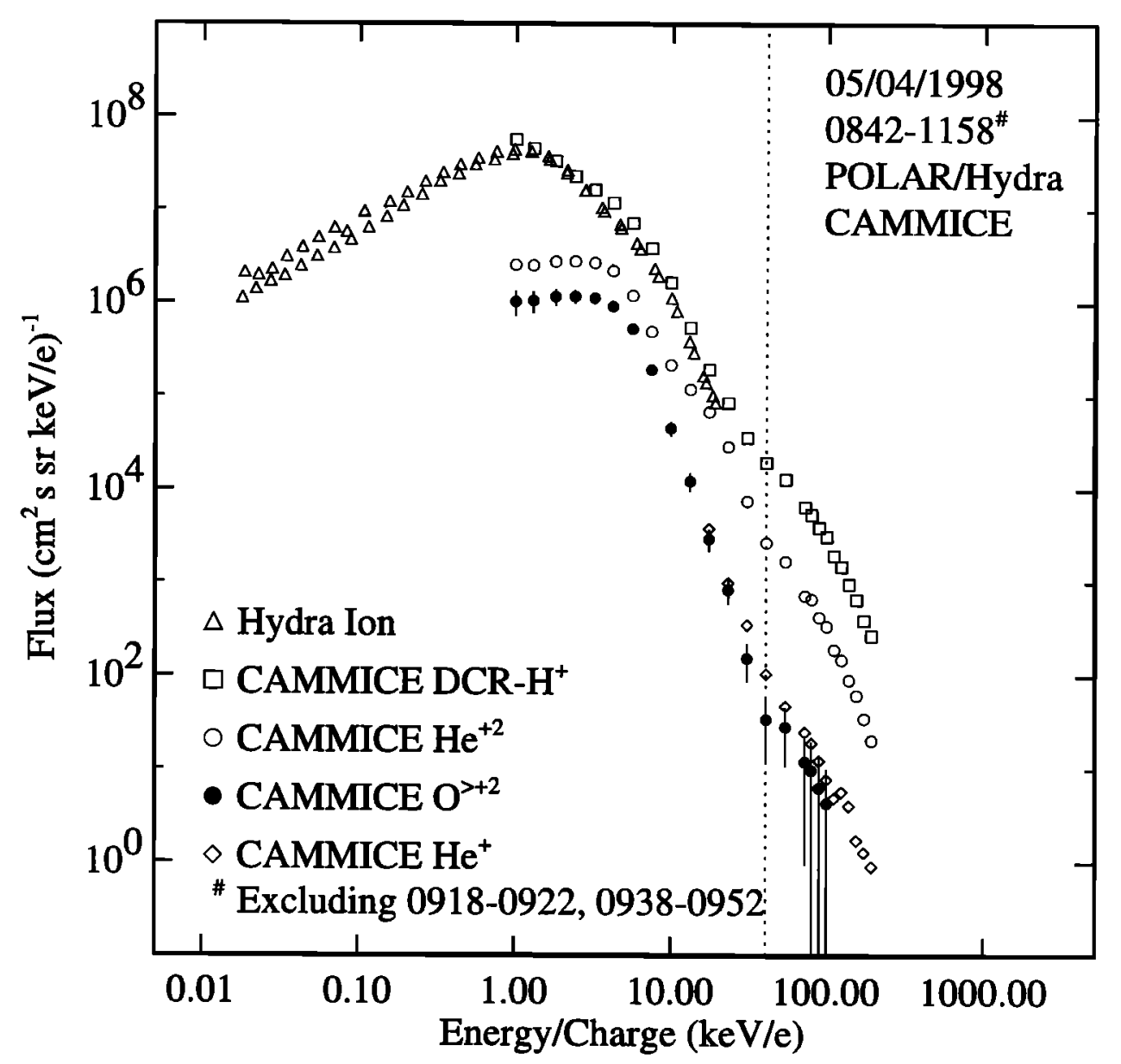

Figure 6. Energy spectra of magnetosheath $\mathrm{H}^{+}, \mathrm{He}^{+2}, \mathrm{O}^{>+2}$, and $\mathrm{He}^{+}$ions from Polar/Hydra and CAMMICE averaged over the interval 0842-1158 UT excluding the solar wind intervals. Spectral shapes of the first three species are similar, ordered by energy per charge. They are nearly identical for $\mathrm{H}^{+}$and $\mathrm{He}^{+2}$ at energies above $20 \mathrm{keV} / e$. Differences in $\mathrm{O}^{>+2}$ are due to low count rate as shown by the error bars. These three spectra are continuous with a common spectral break approximately at the energy channel of $41.1 \mathrm{keV} / e$ (vertical dotted line). Energetic $\mathrm{He}^{+}$ions show a slightly harder spectrum with a less obvious spectral break.

in the two instruments. In this plot format, spectral shapes for the first three ion species above are similar; in particular, they are nearly identical for $\mathrm{H}^{+}$and $\mathrm{He}^{+2}$ for energies above $20 \mathrm{keV} / e$. The differences in $\mathrm{O}^{>+2}$ may be attributed to the low counting statistics which get worse with increasing energy. Nevertheless, all three spectra are continuous with a spectral break at about the detector energy channel of $41.1 \mathrm{keV} / e$ where the spectral curves start to deviate from their common higher energy power law tail. This break is most clear when spectra are viewed from the highest energy toward lower energy. These spectra resemble those upstream and downstream from the quasi-parallel bow shock (cf. Figures 3 and 4 of Möbius et al. [1987] and Figure 2 of Ellison et al. [1990]). Although there are substantial intensity variations for these ions during this magnetosheath interval, their spectral shapes remain similar.

Unlike the above three ion species, $\mathrm{He}^{+}$has a somewhat different spectral shape. This spectrum is slightly harder and does not show an obvious spectral break.
This suggests $\mathrm{He}^{+}$may have a source different from those of the other three ion species.

A cross-correlation analysis of integrated $\mathrm{H}^{+}$flux with the IMF cone angle $\theta_{B x}$ (the angle between the IMF and Sun-Earth line) was performed to examine the hypothesis that magnetosheath energetic ions are regulated by the bow shock geometry permitted by the cone angle which is used as a proxy of $\theta_{B n}$ (see the Discussion section below). Figure 7 a presents the $\mathrm{H}^{+}$flux integrated $41.1-193.4 \mathrm{keV} / e$ at the highest time resolution from 0840 to $1200 \mathrm{UT}$ and $\theta_{B x}$ offset by $36 \mathrm{~min}$. Both quantities show large temporal variations with the maximum to minimum flux ratio $\sim 100$ and $\theta_{B_{x}}$ ranging $\sim 10^{\circ}-90^{\circ}$. In addition, $\mathrm{H}^{+}$flux and $\theta_{B x}$ are strongly anticorrelated with a correlation coefficient of -0.82 .

The correlation coefficient for the energetic ion flux and $\theta_{B x}$ during this magnetosheath interval depends on the time lag assumed for $\theta_{B x}$ ranging from 0 to 60 min with an 1-min increment as shown in Figure 7b. Starting at 0 -min toward $60-\mathrm{min}$ lag, the correlation 

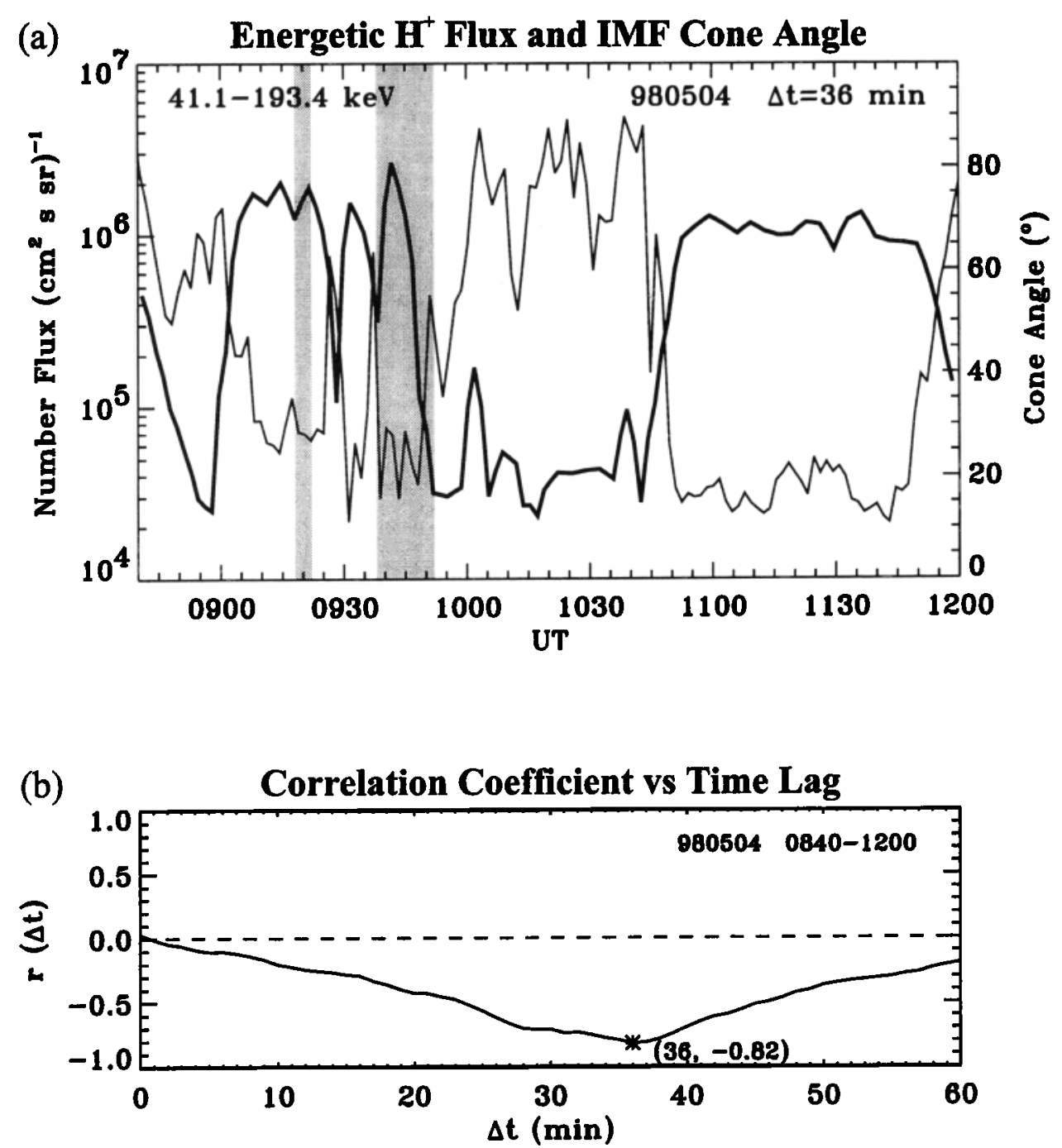

Figure 7. (a) Energetic $(41.1 \leq E \leq 193.4 \mathrm{keV}) \mathrm{H}^{+}$ion flux (thick line) from Polar/CAMMICE in the interval 0840-1200 UT and $\theta_{B x}$ (thin line) from Wind/MFI offset $36 \mathrm{~min}$. Both show large temporal variations and are strongly anticorrelated. (b) Correlation coefficient as a function of the time lag assumed for $\theta_{B x}$. As the lag increases from 0 to $60 \mathrm{~min}$, the coefficient decreases from $\sim 0$, reaches the peak at -0.82 for a lag of $36 \mathrm{~min}$, and then monotonically increases toward 0 . Note that the shaded intervals are excluded in the calculation of the correlation coefficient.

coefficient decreases from $\sim 0$, reaches its peak at -0.82 for a lag of $36 \mathrm{~min}$, and then monotonically increases toward 0 . This 36-min lag associated with the optimal correlation is consistent with the estimated solar wind propagation time and other time estimates (see discussions below).

Ions of three species, namely $\mathrm{H}^{+}, \mathrm{He}^{+2}$, and $\mathrm{O}^{>+2}$, respond similarly at energies above and below their common spectral break point. Correlation coefficients of the integrated magnetosheath ion flux with $\theta_{B x}$ as a function of the time lag $(0-60 \mathrm{~min})$ for these ions are presented in Plate 2. For comparison, results of correlation analysis for $\mathrm{He}^{+}$are also included in Plate 2. The energy range of integration is from $E_{1}$ to $E_{2}$, where $E_{1}$ steps through the detector energy channels from 1.0 ( 17.5 for $\mathrm{He}^{+}$) to $100.1 \mathrm{keV} / e$ and $E_{2}$ is fixed at the highest channel, $193.4 \mathrm{keV} / e$ for all the species but
$\mathrm{O}^{>+2}$ whose maximum energy is $100.1 \mathrm{keV} / e$. These correlation curves are color coded according to their $E_{1}$ values displayed in the color bar. The first remarkable result is that all the curves at $E_{1} \geq 41.1 \mathrm{keV} / e$ (blue) from Plates $2 \mathrm{a}$ and $2 \mathrm{~b}$ are nearly identical. In addition, these curves have a unique peak correlation coefficient $\sim-0.8$ at a time lag of $\sim 36 \mathrm{~min}$, suggesting that magnetosheath energetic $(\geq 41.1 \mathrm{keV} / e) \mathrm{H}^{+}$and $\mathrm{He}^{+2}$ ion fluxes are strongly anticorrelated with $\theta_{B x}$.

The correlation curves for $\mathrm{H}^{+}$and $\mathrm{He}^{+2}$ begin to change drastically by including ion fluxes from one or two energy channels below $41.1 \mathrm{keV} / e$. They become much flatter and closer to the line of null correlation as shown by the green curves in panels $a$ and $b$. This result suggests a critical energy for the anticorrelation between the magnetosheath energetic ion flux and $\theta_{B x}$. As $E_{1}$ continues to decrease toward $1 \mathrm{keV} / e$, the correlation 


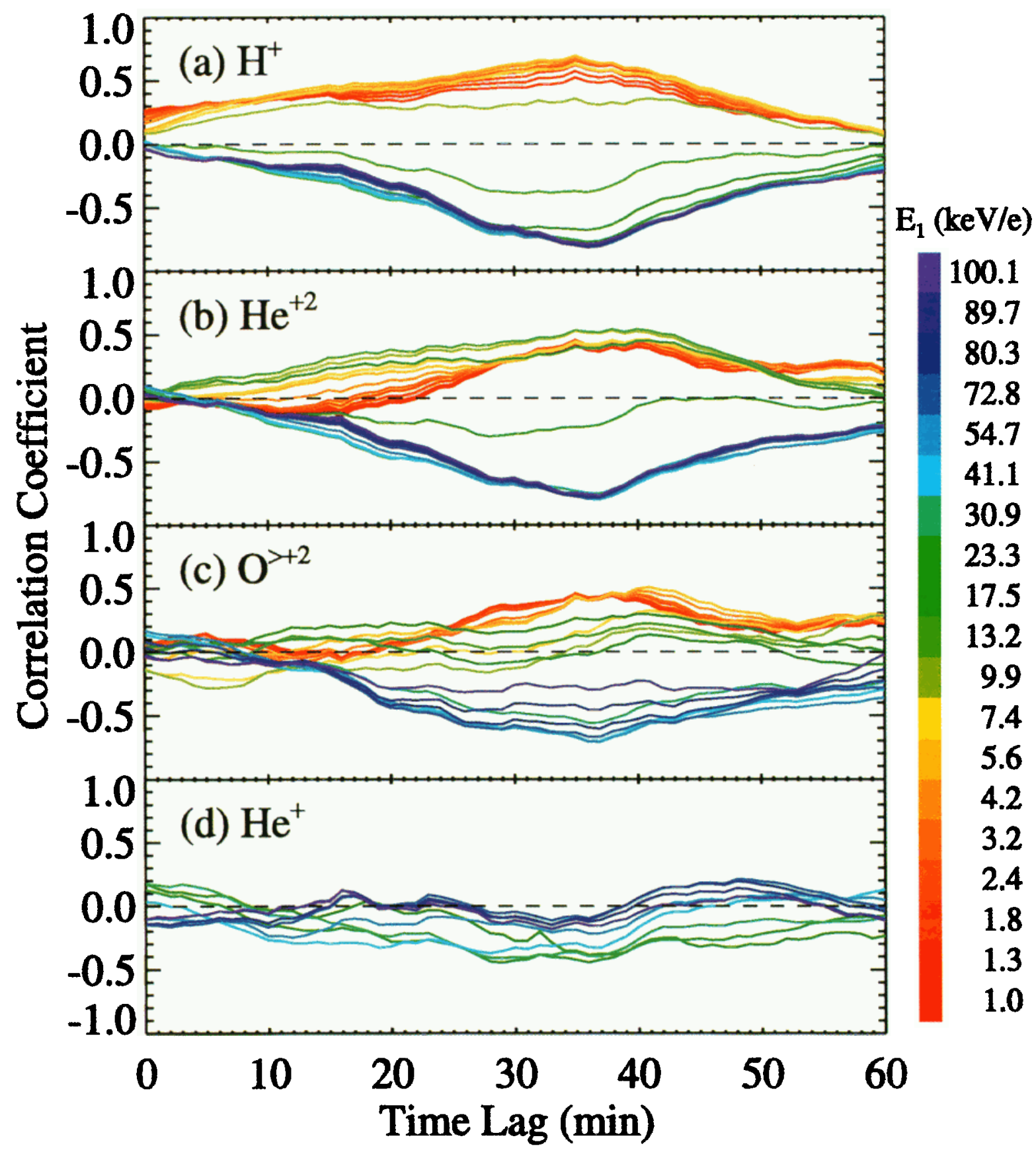

Plate 2. Correlation coefficient curves of the magnetosheath ion flux $\left(E_{1} \leq E \leq E_{2}\right)$ and $\theta_{B x}$ for three ion species, (a) $\mathrm{H}^{+}$, (b) $\mathrm{He}^{+2}$, (c) $\mathrm{O}^{>+2}$, and (d) $\mathrm{He}^{+}$. Curves are color coded according to their $E_{1}$ 's given in the color bar with $E_{2}$ equal to $193.4 \mathrm{keV} / e$ for $\mathrm{H}^{+}, \mathrm{He}^{+2}$, and $\mathrm{He}^{+}$and $101.1 \mathrm{keV} / e$ for $\mathrm{O}^{>+2}$. All the curves with $E_{1} \geq 41.1 \mathrm{keV} / e$ (blue) in Plates $2 \mathrm{a}$ and $2 \mathrm{~b}$ are nearly identical, showing a strong anticorrelation between the $\mathrm{H}^{+}, \mathrm{He}^{+2}$ ion flux and $\theta_{B x}$ offset $\sim 36$ min with the peak correlation coefficient $\sim-0.8$. Below $41.1 \mathrm{keV} / e$, curves sharply shift toward weakly positive correlation (red). Similar trends also appear in $\mathrm{O}^{>+2}$. Correlation curves for $\mathrm{He}^{+}$show a trend opposite to the other three species. Literally, no correlation is found between energetic $\mathrm{He}^{+}$ion flux and $\theta_{B x}$. 
curves for $\mathrm{H}^{+}$and $\mathrm{He}^{+2}$ begin to show some positive correlation and then move toward weak correlation as indicated by the yellow and red curves. Because energetic $\mathrm{O}^{>+2}$ ions have very low count rates, correlation curves are rather irregular as shown in panel c. Nevertheless, these curves show a trend similar to those for $\mathrm{H}^{+}$and $\mathrm{He}^{+2}$. Furthermore, at lower energies $\mathrm{O}^{>+2}$ ions have sufficient statistics to yield correlation coefficient curves with more significance. These curves are nearly identical to those of $\mathrm{He}^{+2}$ at the same energies (cf. red curves in Plates $2 b$ and $2 c$ ).

Correlation coefficient curves for $\mathrm{He}^{+}$illustrate a completely different picture. As shown by the green curves in panel $\mathrm{d}, \mathrm{He}^{+}$flux at lower energies is only slightly anticorrelated with $\theta_{B x}$. At higher energies (blue curves) there is nearly no correlation between the ion flux and $\theta_{B x}$. These results demonstrate a trend opposite to the one for the other three ion species.

To demonstrate the strong energy dependence in the correlation coefficient, the peak correlation coefficient $r_{o}$ is plotted against $E_{1}$ for each ion species as shown in Figure 8a. There are two types of curves, one for three ion species $\mathrm{H}^{+}, \mathrm{He}^{+2}$, and $\mathrm{O}^{>+2}$ and the other for $\mathrm{He}^{+}$. In the first type it is clear that a critical energy $E_{c}$ has to be reached for ion flux to be anticorrelated with $\theta_{B x}$. It is the energy at which correlation curves start to deviate from the plateau at high energies. This energy approximately occurs at the detector energy channel of $41.1 \mathrm{keV} / \mathrm{e}$ for all three ion species and happens to be the same energy channel at the spectral break suggested in Figure 6. Furthermore, $r_{o}$ values are nearly constant for $E_{1}$ above $E_{c}, \sim-0.82$ for both $\mathrm{H}^{+}$and $\mathrm{He}^{+2}$ and $\sim-0.71$ for $\mathrm{O}^{>+2}$. However, when $E_{1}$ is above $70 \mathrm{keV} / e, r_{o}$ begins to move toward 0 due to poorer counting statistics. This trend is more obvious for higher-energy $\mathrm{O}^{>+2}$ since their fluxes are nearly close to the background value. Nonetheless, the sharp transition from strong anticorrelation to moderate positive correlation just below $E_{c}$ is vividly illustrated. The curve of the second type, on the other hand, shows a completely different result. It clearly demonstrates a trend opposite to the curves for the other three species.

The time lag $\Delta t_{o}$ associated with the peak correlation can be quite different for different energies as shown in Figure 8b. However, when $r_{o}$ is very small $(<-0.7), \Delta t_{o}$ is nearly the same for $\mathrm{H}^{+}, \mathrm{He}^{+2}$, and $\mathrm{O}^{>+2}$ ions at $\sim 36 \mathrm{~min}$. This common $\Delta t_{o}$ suggests a unique signal transmission time independent of ion energy and species. In contrast, $\Delta t_{o}$ for $\mathrm{He}^{+}$varies with the ion energy and deviates further away from the common lag for the other three species. Results of the cross-correlation analysis for the three ion species have demonstrated that magnetosheath energetic ions with energy above the spectral break are strongly anticorrelated with $\theta_{B x}$ offset by a lag consistent with the solar wind propagation time and other time estimates that are addressed in the discussion section. Results of $r_{o}$ and $\Delta t_{o}$ for lower-energy $\left(E_{1}<10 \mathrm{keV} / e\right)$ ions shown in Figure 8, however, can only indicate average results for lower-energy ions and are discussed more later.

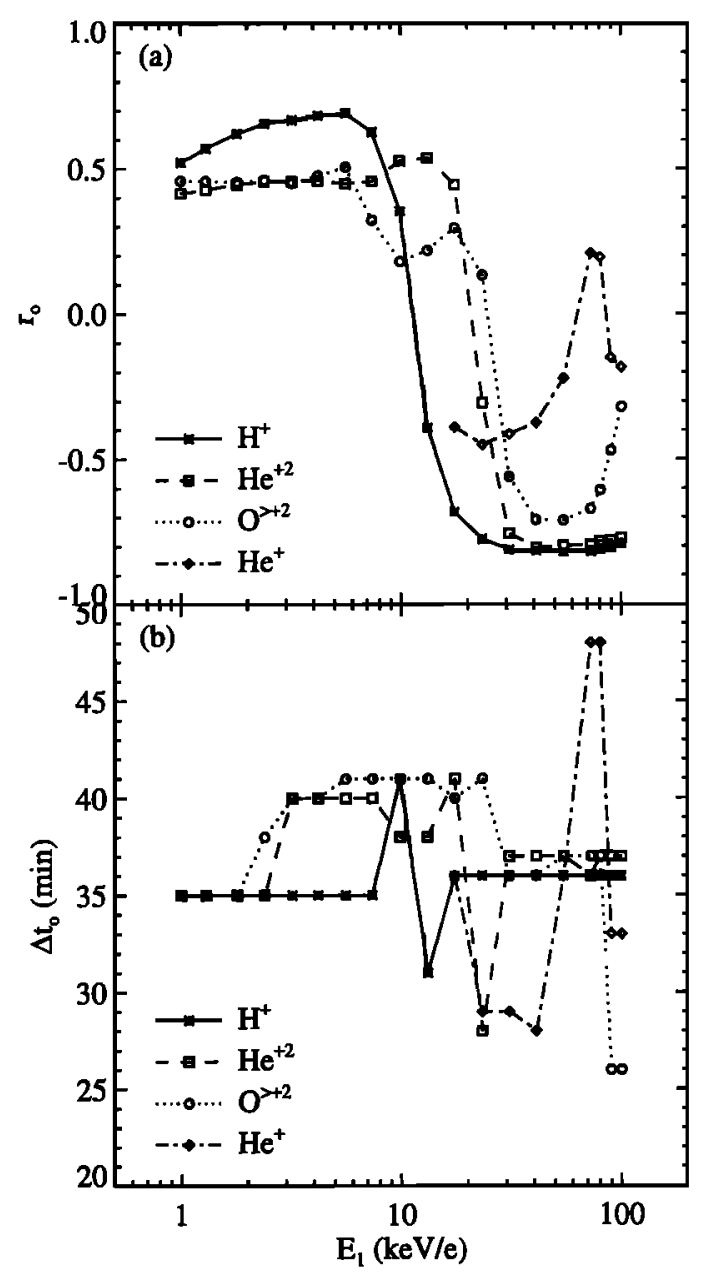

Figure 8. (a) Peak correlation coefficient $r_{o}$ and (b) the associated time lag $\Delta t_{o}$ as a function of energy $E_{1}$ for four ion species. Ion fluxes for $\mathrm{H}^{+}, \mathrm{He}^{+2}$, and $\mathrm{O}^{>+2}$ with energy above a critical energy, $E_{c}=41.1 \mathrm{keV} / \mathrm{e}$, are anticorrelated with $\theta_{B x}$ offset $\Delta t_{o} \sim 36$ min. Below $E_{c}$ this anticorrelation disappears. Energetic $\mathrm{He}^{+}$ fluxes, on the other hand, are not correlated with $\theta_{B x}$.

Since the observed magnetosheath energetic $\mathrm{H}^{+}$, $\mathrm{He}^{+2}$, and $\mathrm{O}^{>+2}$ ion fluxes are controlled by $\theta_{B x}$, one would expect they flow antisunward away from the bow shock. In this storm event the magnetosheath magnetic field was most of the time close to the Polar's spin axis so that CAMMICE detector only covered a limited range of pitch angle around $90^{\circ}$. However, during the interval from $\sim 0850$ to $1000 \mathrm{UT}$, CAMMICE produced a nearly full coverage of pitch angle for energetic ions and can then deduce their average flow direction. Figure 9 presents the normalized angular distribution of energetic ion flux at the highest time resolution for $\mathrm{H}^{+}, \mathrm{He}^{+2}$ (both $41.1 \leq E \leq 193.4 \mathrm{keV} / e$ ), and $\mathrm{O}^{>+2}$ $(41.1 \leq E \leq 101.1 \mathrm{keV} / e)$ in the spacecraft frame for three different intervals in the magnetosheath. Because the plasma flow speed measured by Hydra in the Polar spin plane is $\sim 300 \mathrm{~km} / \mathrm{s}$ which is much smaller than the speed of the lowest-energy ions (2800 and $2000 \mathrm{~km} / \mathrm{s}$ for $41.1-\mathrm{keV} / e \mathrm{H}^{+}$and $\mathrm{He}^{+2}$, respectively), the maximum pitch angle correction for ion distribution in the 


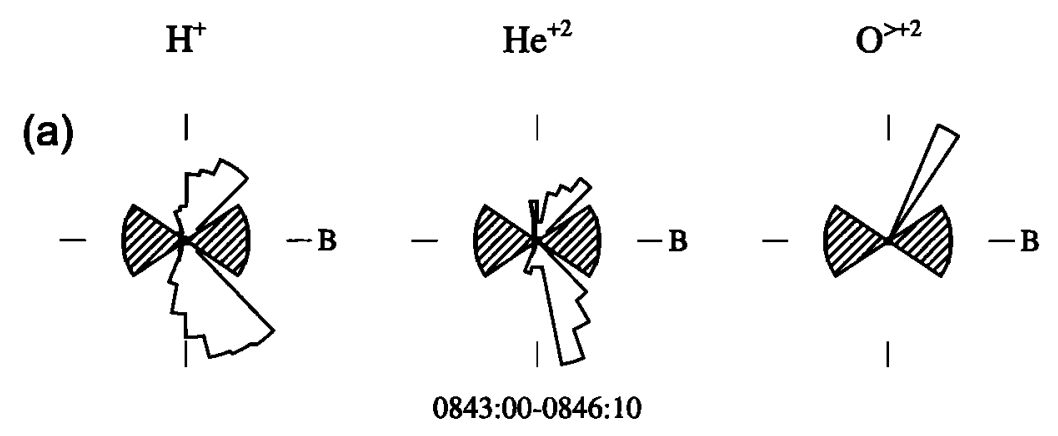

(b)
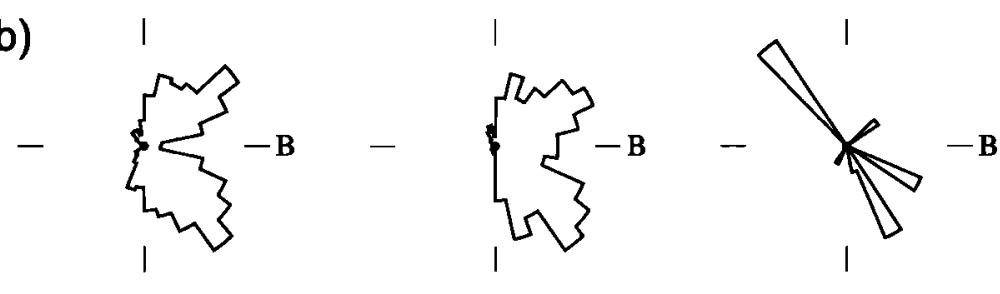

0904:00-0907:08

(c)

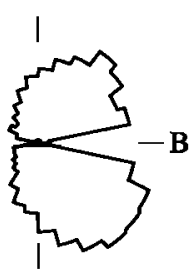

।

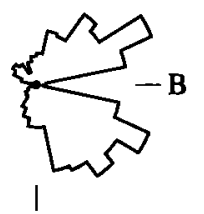

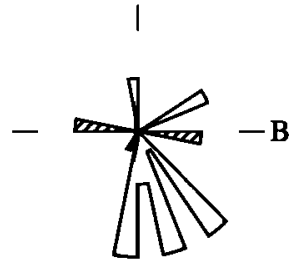

0914:00-0917:15

Figure 9. Normalized angular distribution of energetic ion flux for $\mathrm{H}^{+}, \mathrm{He}^{+2}$ (both $41.1 \leq E \leq$ $193.4 \mathrm{keV} / e)$, and $\mathrm{O}^{>+2}(41.1 \leq E \leq 101.1 \mathrm{keV} / e)$ in the spacecraft frame for three intervals in the magnetosheath, (a) near the magnetopause boundary layer, (b) in the magnetosheath proper, and (c) near the bow shock. $\mathrm{H}^{+}$and $\mathrm{He}^{+2}$ ions are streaming along the magnetic field direction. The hatched areas in Figures 9a and 9c indicate pitch angles that were not sampled. Energetic $\mathrm{O}^{>+2}$ ions show a trend consistent with the other two species although their statistics are poorer.

rest frame of plasma is less than $9^{\circ}$, smaller than the maximum pitch angle width $\left(11.25^{\circ}\right)$ sampled by the CAMMICE detector in each measurement. Therefore ion distributions in the rest frame of plasma are expected to be similar to those in the spacecraft frame.

As shown in Figures $9 \mathrm{~b}$ and $9 \mathrm{c}$, almost all the $\mathrm{H}^{+}$and $\mathrm{He}^{+2}$ in the magnetosheath proper and near the bow shock have pitch angles less than $90^{\circ}$ with the maximum flux occurring at $\sim 45^{\circ}$ pitch angle. Therefore these ions show a strong flow along the magnetic field direction. During the interval covered in Figure 9a, Polar was in the magnetosheath close to the magnetopause and energetic ions with pitch angles within $30^{\circ}$ to the magnetic field line were not sampled by CAMMICE. Nevertheless, the observed partial angular distributions for $\mathrm{H}^{+}$ and $\mathrm{He}^{+2}$ in this region are consistent with those observed in the magnetosheath proper and near the bow shock. As to energetic $\mathrm{O}^{>+2}$ ions, they show a trend similar to those of the other two species although their statistics are poorer.

Within the above magnetosheath interval (0850-1000 UT), the angular distributions of energetic ions are basically the same as those presented in Figure 9, showing a predominant flow along the magnetic field direction. Meanwhile the magnetosheath magnetic field $B_{x}$ component turned negative and $B_{z}$ turned positive after 0840 UT and maintained the same polarity until $\sim 1002$ UT as shown in Figure 4. Therefore the observed magnetosheath energetic ions during the above interval were streaming antisunward and northward away from the bow shock.

\section{Discussion}

In the previous section we have presented plasma and magnetic field measurements from the NASA GGS/Polar and Wind satellites for the May 4, 1998, storm event. Here we discuss the possible origin of the observed magnetosheath energetic ions. While two major sources are the solar wind and magnetospheric plasmas, the mechanism of acceleration and transport can be quite different for each of the sources. Energetic magnetospheric ions in the plasma sheet on closed field lines can leak into the magnetosheath [e.g., Croley et al., 1986; Sibeck et al., 1987]. Their intensity is more related to geomagnetic activity [e.g., Christon et al., 1994]. Energetic ions 
previously on closed field lines can escape the magnetosphere into the magnetosheath along open field lines either by gradient and curvature drift across field lines or magnetic reconnection [e.g., Scholer et al., 1981]. Solar energetic particles can also directly contribute to the magnetosheath energetic ions. Diffuse ions accelerated at the quasi-parallel bow shock can be another possibility [e.g., West and Buck, 1976; Fuselier et al., 1991].

Because there are two types of energetic ions observed in the magnetosheath, one for $\mathrm{H}^{+}, \mathrm{He}^{+2}$, and $\mathrm{O}^{>+2}$ and the other for $\mathrm{He}^{+}$. Each type shows distinct features as presented in the previous section. This suggests the acceleration region for $\mathrm{He}^{+}$ions is different from the one(s) for $\mathrm{H}^{+}, \mathrm{He}^{+2}$, and $\mathrm{O}^{>+2}$ ions. The discussions below apply to the first type for the three ion species unless stated otherwise.

The composition of the observed magnetosheath energetic ions $\left(\mathrm{He}^{+2}, \mathrm{O}^{>+2}\right)$ suggest they are ultimately from the solar wind not from the ionosphere. It is a question whether $\mathrm{keV}$ ions of solar wind origin entered the magnetosphere, convected to the magnetotail, were accelerated there and injected to the dayside, and then escaped the magnetosphere. Because the dayside plasma sheet/LLBL and magnetosheath traversed by Polar during this event show distinct energetic ion composition, leakage of magnetospheric ions is not likely the main cause of the magnetosheath energetic ions. In addition, Polar was on magnetosheath field lines which were most of the time connected to the solar wind, not the open magnetosphere (cusp, mantle, lobe, etc.). Escape of energetic ions along open field lines is unlikely an explanation for the observations. Most importantly, a magnetospheric source would not explain the magnetosheath energetic ion flux of solar wind origin being anticorrelated with the IMF cone angle $\left(\theta_{B x}\right)$.

Solar energetic ions observed by Wind are much less intense than the magnetosheath energetic ions during this event. Results of cross-correlation analysis reveal that ion fluxes in the above two regions are slightly anticorrelated. Therefore solar energetic ions are not a direct source of magnetosheath energetic ions. However, an acceleration region at the bow shock can account for ions accelerated to hundreds of $\mathrm{keV}$. It is noted that energetic ion spectra downstream from the shock are nearly identical to those in the upstream wave region (see, for example, Figure 10 of West and Buck [1976]). Ions undergo first-order Fermi acceleration by scattering back and forth across the quasi-parallel bow shock in the turbulent regions upstream and downstream from the shock. This mechanism is most efficient for highly charged ions [Lee, 1982]. The magnetosheath energetic ion spectra observed by Polar have shown such a property.

Results of the cross-correlation analysis for the magnetosheath ion flux and $\theta_{B x}$ are very striking. In order to lower the statistical error especially for higher-energy

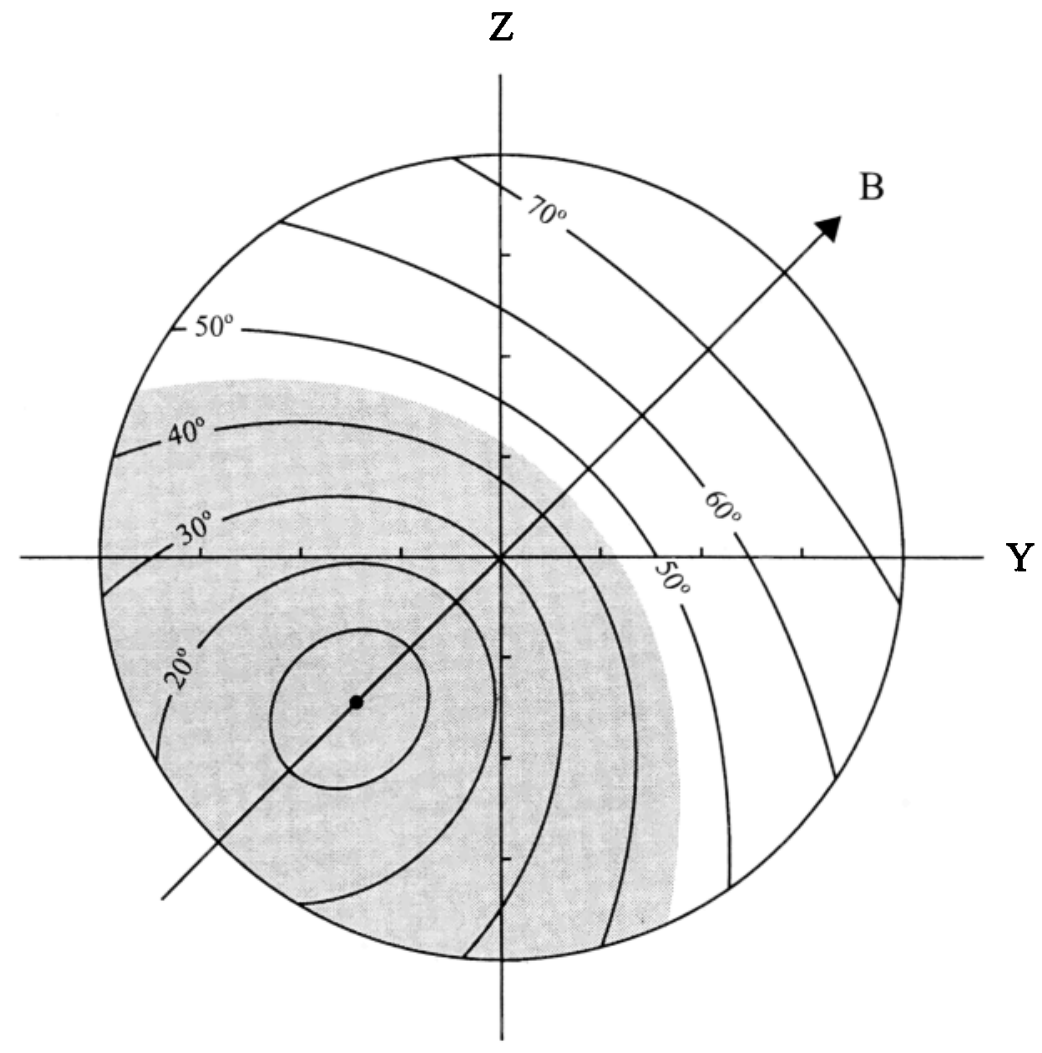

Figure 10. Projection of the dayside bow shock surface onto the $y-z$ plane using the Fairfield [1971] model. Contours of constant $\theta_{B n}$ are calculated for IMF $\left(B_{x}, B_{y}, B_{z}\right)=(-13.3,5.5,5.5)$ nT with $\theta_{B x}=30.3^{\circ}$. The shaded region is the quasi-parallel shock located at the most part of the southern hemisphere and part of the northern hemisphere. 
ions, we have done this analysis using the integrated ion number flux instead of the differential number flux at each MICS energy channel. Because of the steep drop in the energetic tail of the ion spectra, ion flux detected at the lowest energy channel in the integration energy range contributes most to the total flux. Therefore the integrated flux for ion energy above $\sim 10 \mathrm{keV} / e$ truly reflects the behavior of the differential flux at the lowest energy as demonstrated by the sharp transition in Figure 8. In fact, the correlation coefficient curves for ion differential number flux at energy above $\sim 10 \mathrm{keV} / e$ are very similar to the corresponding ones for the integrated flux presented in Plate 2. On the other hand, for ion energy below $10 \mathrm{keV} / e$ the ion differential energy flux at the lowest energy of the integration does not dominate the total flux because the ion flux at this energy is not large enough to compensate the reduced energy in the integration. Thus results of correlation analysis including ions below $\sim 10 \mathrm{keV} / e$ only represent average results, rather than results for ions detected at the lowest energy channel within the integration. The correlation curves for the differential ion flux at energy below $10 \mathrm{keV} / e$ become more erratic showing a trend toward null correlation with decreasing energy, unlike the smooth, asymptotic behavior shown in Figure 8. This result of little correlation is expected because the intensity of the keV solar wind ions at the bow shock is not related to the IMF or shock geometry.

The most remarkable feature in the results of the cross-correlation analysis is that the correlation coefficient is energy dependent. Correlation coefficients as a function of ion energy show a sharp transition at the same energy (precisely energy per charge) for all three ion species. Above this critical energy ion fluxes are strongly anticorrelated with $\theta_{B x}$ and essentially uncorrelated for energies immediately below it. It is even more striking that this critical energy is identical to the energy at the spectral break of the ion spectra. All the above results are consistent with the Fermi acceleration process since it is responsible for the energetic ions above the spectral break and creating the break [e.g., Lee, 1982]. This process takes place at the quasi-parallel bow shock.

Statistical studies show that probability of enhanced energetic ion events upstream and downstream from the bow shock increases as $\theta_{B n}$ of the field line being sampled decreases, and vice versa [e.g., Crooker et al., 1981; Mitchell and Roelof, 1983]. In principle an appropriate $\theta_{B n}$ should be used in the cross-correlation analysis. Since the observed magnetosheath energetic ions are extremely energetic, they are guided more or less by the magnetic field lines not the flow streamlines in the subsonic medium [e.g., Bonifazi and Moreno, 1981; Mitchell and Roelof, 1983]. For example, a $41.1 \mathrm{keV} / e \mathrm{H}^{+}$ion with a pitch angle of $45^{\circ}$, the velocity component parallel to the magnetic field is $\sim 1900 \mathrm{~km} / \mathrm{s}$ which is more than 4 times of the $E \times B$ drift speed observed in the magnetosheath. Because the associated $\theta_{B n}$ where the magnetosheath magnetic field line through Polar intersets the bow shock is not readily known, $\theta_{B x}$ is used as a proxy for $\theta_{B n}$ in this study. Furthermore, $\theta_{B_{x}}$ and the angle between the IMF $B_{y}$ and $B_{z}$ components would completely determine the bow shock magnetic geometry.

Magnetosheath energetic ion fluxes are enhanced in two intervals, $\sim 0900-1000$ and $\sim 1100-1200$ UT. Figure 10 shows the average dayside bow shock surface projected onto the $y-z$ plane for the first interval. This bow shock surface is based on the Fairfield model [Fairfield, 1971]. For the average IMF associated with this interval, $(-13.3,5.5,5.5) \mathrm{nT}, \theta_{B x}$ is $30.3^{\circ}$ and the quasiparallel shock as shown by the shaded region covers the major portion of the southern hemisphere and part of the northern hemisphere of the dayside bow shock surface. During the second interval, IMF $B_{x}$ and $B_{z}$ components reverse the orientation and $\theta_{B x}$ is further reduced to $\sim 10^{\circ}$. The quasi-parallel bow shock switches to the east side and further expands, covering a great portion of the southern hemisphere and most of the northern hemisphere of the dayside bow shock surface. As for the interval of weak magnetosheath energetic ion fluxes, $\sim 1000-1100 \mathrm{UT}, \theta_{B x}$ is $\sim 80^{\circ}$ and nearly the entire dayside bow shock surface is quasi-perpendicular. The quasi-parallel shock in this case occupies only a small portion of the dusk flank for IMF $B_{y}$ positive.

The majority of magnetosheath energetic ions observed by Polar have pitch angles $\sim 45^{\circ}$ (Figure 9 ). Thus almost all the energetic ions with the exception of those with pitch angles nearly $90^{\circ}$, would be guided by the magnetic field lines. During the first interval of the enhanced magnetosheath energetic ion flux, 0900-1000 UT, both IMF and magnetosheath magnetic field have a negative $B_{x}$ and positive $B_{z}$ component. Such an IMF condition is commonly seen during CEP events [Chang et al., 1998]. On the basis of the general solar wind flow and magnetosheath flow [Spreiter and Stahara, 1985] the most likely scenario for the magnetic field topology is illustrated in Figure 11. Polar is on magnetosheath magnetic field lines (e.g., field line a) that are connected to the quasi-parallel bow shock (cf. Figure 10). Energetic ions produced at the shock follow field lines, reach Polar and are detected by the CAMMICE instrument. This magnetic topology also appears in the global hybrid simulations results for this IMF boundary condition (N. Omidi, private communication, 1999). For the second interval of intense energetic ion flux, magnetosheath magnetic field $B_{x}$ and $B_{z}$ components reverse their sign, but the magnetic topology remains similar. Polar is again on a field line connected to the quasi-parallel bow shock and observes intense energetic ions. On the other hand, between the two intervals of enhanced energetic ion flux, $\theta_{B x}$ is very large and $\mathrm{Po}^{-}$ lar is on field lines connected to the quasi-perpendicular shock and detects very weak energetic ion flux.

Because the observed energetic ions have a very high speed parallel to the magnetic field line, it takes less than $1 \mathrm{~min}$ for them to travel from the quasi-parallel bow shock to the Polar location in the magnetosheath. The solar wind propagation time from the Wind satellite to the bow shock is $\sim 29 \mathrm{~min}$ in the interval se- 


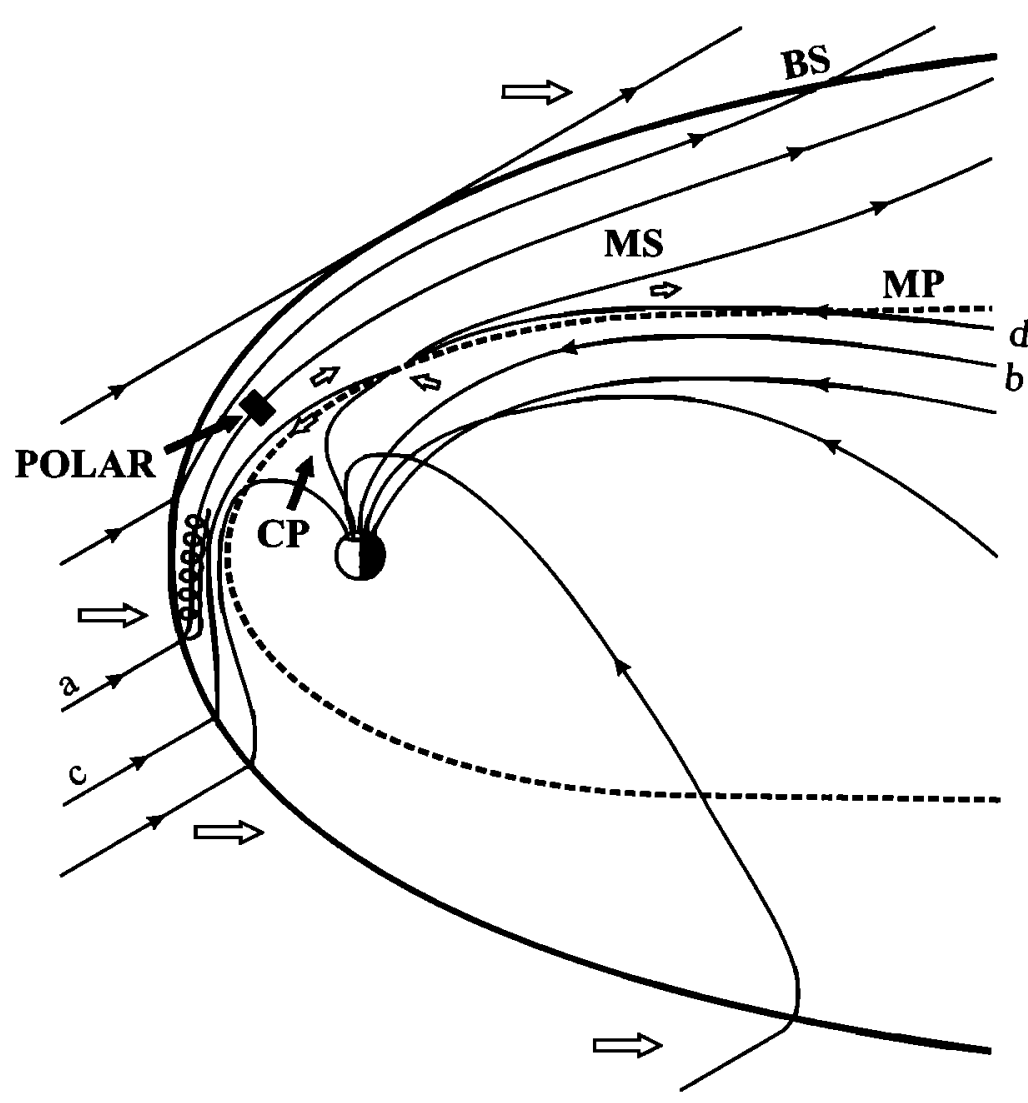

Figure 11. Schematic of the geospace under a northward and antisunward IMF condition, showing the bow shock (BS), magnetosheath (MS), magnetopause (MP), cusp (CP), merging site, and the Polar location. Polar is in the magnetosheath upstream from the cusp and on magnetic field lines (e.g., field line a) connected to the quasi-parallel bow shock, observing the tailwardstreaming energetic ions that are accelerated at the quasi-parallel shock. The magnetosheath field orientation is northward and antisunward in favor of high-latitude reconnection, poleward of the cusp and energetic magnetosheath ions can then directly enter the cusp along the interconnected field lines (e.g., field line c).

lected for the correlation analysis. However, the associated time lag for the best correlation between the energetic ion flux and $\theta_{B x}$ is $\sim 36 \mathrm{~min}$ that is $\sim 7 \mathrm{~min}$ more than the estimated solar wind propagation time. This time difference can be attributed to the growth time for the energetic ions at the bow shock. As the bow shock geometry changes from quasi-perpendicular to quasi-parallel, a growth time of $\sim 10 \mathrm{~min}$ is required for enhanced 50-200 keV ions [e.g., Scholer et al., 1980; Mitchell and Roelof, 1983]. Taking into account the uncertainty due to the CAMMICE detector sampling time ( $3.3 \mathrm{~min})$, the above time difference is consistent with the early finding of the average growth time.

Unlike the above three ion species, $\mathrm{He}^{+}$fluxes are not correlated with $\theta_{B x}$. Therefore the observed energetic $\mathrm{He}^{+}$ions are not Fermi accelerated at the shock. Since their fluxes are very weak and relatively uniform throughout the midlatitude magnetosheath interval, leakage of magnetospheric ions may account for these ions [e.g., Kudela et al., 1992].

There is little doubt that the majority of magnetosheath energetic ions came from the quasi-parallel bow shock during this event. As discussed above, $\mathrm{He}^{+2}$ ions are a part of the solar wind ions accelerated at the quasi-parallel shock not the leaked magnetospheric ions and then transported to the magnetosheath. The remarkable similarity between the magnetosheath energetic $\mathrm{H}^{+}$and $\mathrm{He}^{+2}$ ions in three aspects, spectral shape, correlation coefficient curves, and angular distribution, suggests that energetic $\mathrm{H}^{+}$ions are likely the solar wind protons accelerated at the quasi-parallel shock and then transported to the magnetosheath just like $\mathrm{He}^{+2}$. Magnetospheric contribution to the magnetosheath energetic ions has to be very minor. The simultaneously enhanced low-frequency waves associated with the magnetosheath energetic ions (Plate 1) are possibly like the energetic ions from the quasi-parallel bow shock as reported before [e.g., Luhmann et al., 1986; Möbius et al., 1987]. When Polar was upstream from the cusp in the magnetosheath and magnetosheath field had a negative $B_{x}$ and positive $B_{z}$, according to the antiparallel merging hypothesis the reconnection site was probably at the high-latitude magnetopause poleward of the cusp [Crooker, 1979]. Magnetosheath field lines would be subsequently interconnected with the cusp field line and energetic ions produced at the quasi-parallel shock 
would then enter the cusp along the interconnected field lines (e.g., field line $\mathrm{c}$ in Figure 11). The present investigation of magnetosheath energetic ions observed by Polar strongly support the hypothesis of bow shock source of CEPs [Chang et al., 1998].

\section{Summary and Conclusions}

We have presented plasma and magnetic field observations from NASA GGS/Polar and Wind satellites during the May 4, 1998, magnetic storm event. Polar was at the dayside magnetosheath in the northern hemisphere and observed energetic (41.1-193.4 keV/e) $\mathrm{H}^{+}$, $\mathrm{He}^{+2}$, and $\mathrm{O}^{>+2}$ ion fluxes with large temporal variations (maximum to minimum flux ratio $\sim 100$ ). Their intensity is controlled by the bow shock magnetic geometry. These intense energetic ions appear to be from the bow shock accelerated ions, not from the magnetosphere based on the following results.

1. Polar observed distinct ion composition $\left(\mathrm{O}^{<+3}\right)$ and different relative abundance of $\mathrm{H}^{+}, \mathrm{He}^{+2}$, and $\mathrm{O}^{>+2}$ ions in the plasma sheet/LLBL and magnetosheath. These magnetosheath energetic ions are of solar wind origin.

2. The magnetosheath ion energy spectra for all three ion species are continuous with a spectral break at $\sim 40 \mathrm{keV} / e$, resembling those at the quasi-parallel bow shock. Spectral shape for energetic $\mathrm{H}^{+}$and $\mathrm{He}^{+2}$ are nearly identical.

3. These magnetosheath energetic ions flow antisunward away from the bow shock along the magnetic field lines. Angular distributions for $\mathrm{H}^{+}$and $\mathrm{He}^{+2}$ are very similar.

4. Correlation coefficient for the magnetosheath ion flux of each of the three ion species and the IMF cone angle $\left(\theta_{B x}\right)$ depends on the ion energy and time lag for $\theta_{B x}$. Ion flux and $\theta_{B x}$ (offset $\sim 36 \mathrm{~min}$ ) are strongly anticorrelated for energies above the spectral break. Below the break energy there is little correlation.

5. Correlation coefficient curves as a function of the time lag peak at $\sim 36$ min for all three ion species. This lag is consistent with the solar wind propagation time estimated by four different methods plus an allowance for the energization time of the energetic ion fluxes at the quasi-parallel bow shock.

6. Correlation coefficient curves for $\mathrm{H}^{+}$and $\mathrm{He}^{+2}$ with energy above the spectral break are nearly identical.

7. When Polar was on field lines connected to the quasi-parallel bow shock, it observed intense energetic. ion fluxes. When it was on field lines connected to the quasi-perpendicular bow shock, few energetic ions were observed.

These results can be easily understood by the firstorder Fermi acceleration process at the quasi-parallel bow shock. While magnetosheath magnetic field was northward and antisunward in favor of the high-latitude reconnection poleward of the cusp, energetic magnetosheath ions upstream from the cusp were streaming tailward in the magnetic field direction. As the field lines convected and were interconnected with the geomagnetic field lines, energetic magnetosheath ions on these field lines would travel along the interconnected field lines into the cusp. These magnetosheath energetic ion observations strongly support the model of the bow shock source of CEPs [Chang et al., 1998]. Follow-up studies of analyzing multisatellite observations at the bow shock and in the cusp, self-consistent global hybrid simulations, and MHD modeling are underway.

Acknowledgments. This research is supported by $\mathrm{Hy}$ dra NASA funding under grant number NAG 52231 and the German support for Hydra under DARA grant 50 OC 89110 . CAMMICE research at Aerospace is supported by Boston University subcontract GC 152040 NGD which is derived from NASA grant NAG5-7677. The work at UCLA was supported by NASA grant NAG5-3171. We thank R. $P$. Lin for providing Wind/3D Plasma data for our correlation studies. We are grateful to R. D. Holdaway, J. Faden, P. Puhl-Quinn, D. D. Morgan, and J. C. Dorelli for Hydra software activities. The present results of the Hydra investigation were made possible by the decade-long hardware efforts of groups led at NASA GSFC by K. Ogilvie, at UNH by $R$. Torbert, at MPAE by A. Korth and at UCSD by W. Fillius.

Janet G. Luhmann thanks Richard McEntire and another referee for their assistance in evaluating this paper.

\section{References}

Asbridge, J. R., S. J. Bame, and J. B. Strong, Outward flow of protons from the Earth's bow shock, J. Geophys. Res., 73, 5777, 1968.

Asbridge, J. R., S. J. Bame, J. T. Gosling, G. Paschmann, and N. Sckopke. Energetic plasma ions within Earth's magnetosheath, Geophys. Res. Lett., 5, 953, 1978.

Blake, J. B., et al., CEPPAD comprehensive energetic particle and pitch angle distribution experiment on POLAR, Space Sci. Rev., 71, 531, 1995.

Bonifazi, C., and G. Moreno, Reflected and diffuse ions backstreaming from the Earth's bow shock, J. Geophys. Res., 86, 4405, 1981.

Chang, S.-W.. et al., Cusp energetic ions: A bow shock source, Geophys. Res. Lett., 25, 3729, 1998.

Christon. S. P.. D. C. Hamilton, G. Gloeckler, T. E. Eastman. and F. M. Ipavich, High charge state carbon and oxygen ions in Earth's equatorial quasi-trapping region $J$. Geophys. Res., 99, 13.465, 1994.

Croley. D. R., Jr.. J. F. Fennell, and B. G. Ledley, Observation of reconnection phenomena at synchronous orbit, $J$. Geophys. Res., 91, 4321, 1986.

Crooker. N. R., Dayside merging and cusp geometry, J. Geophys. Res., 84, 951, 1979.

Crooker, N. R., T. E. Eastman, L. A. Frank, E. J. Smith, and C. T. Russell, Energetic magnetosheath ions and the interplanetary magnetic field orientation, $J$. Geophys. Res., $86,4455,1981$.

Ellison, D. C.. Shock acceleration of diffuse ions at the Earth's bow shock: Acceleration efficiency and $A / Z$ enhancement. J. Geophys. Res., 90, 29, 1985.

Ellison. D. C., E. Möbius, and G. Paschmann, Particle injection and acceleration at Earth's bow shock: Comparison of upstream and downstream events, Astrophys. J., 352, $376,1990$.

Fairfield, D. H., Average and unusual locations of the Earth's magnetopause and bow shock, J. Geophys. Res., $76,6700,1971$. 
Fuselier, S. A., Energetic magnetospheric protons in the plasma depletion layer. J. Geophys. Res., 97, 13.759, 1992.

Fuselier, S. A., A comparison of energetic ions in the plasma depletion layer and the quasi-parallel magnetosheath. $J$. Geophys. Res., 99, 5855, 1994.

Fuselier, S. A., D. M. Klumpar, and E. G. Shelley, On the origins of energetic ions in the Earth's dayside magnetosheath, J. Geophys. Res., 96, 47, 1991.

Fuselier, S. A., M. F. Thomsen, F. M. Ipavich, and W. K. $\mathrm{H}$. Schmidt, Suprathermal $\mathrm{He}^{2+}$ in the Earth's foreshock region, J. Geophys. Res., 100, 17.107, 1995.

Gosling, J. T., M. F. Thomsen, S. J. Bame, and C. T. Russell, On the source of diffuse, suprathermal ions observed in the vicinity of the Earth's bow shock, J. Geophys. Res., 94, 3555. 1989.

Greenstadt, E. W., Oblique, parallel, and quasi-parallel morphology of collisionless shocks, in Collisionless Shocks in the Heliosphere: Reviews of Current Research, Geophys. Monogr. Ser., vol. 35, edited by B. T. Tsurutani and R. G. Stone, p. 253, AGU, Washington, D. C., 1985.

Greenstadt, E. W., C. T. Russell, and M. Hoppe. Magnetic field orientation and suprathermal ion streams in the Earth's foreshock, J. Geophys. Res., 85, 3473, 1980.

Hones, E. W., Jr., S.-I. Akasofu, S. J. Bame, and S. Singer, Outflow of plasma from the magnetotail into the magnetosheath, J. Geophys. Res., 77, 6688, 1972.

Ipavich, F. M., A. B. Galvin, G. Gloeckler, M. Scholer, and D. Hovestadt. A statistical survey of ions observed upstream of the Earth's bow shock: Energy spectra, composition, and spatial variation, J. Geophys. Res., 86, 4337, 1981.

Kudela, K., D. G. Sibeck, M. Slivka, S. Fischer, V. N. Lutsenko, and D. Venkatesan, Energetic electrons and ions in the magnetosheath at low and medium latitudes: Prognoz 10 data, J. Geophys. Res., 97, 14,849, 1992.

Lee, M. A., Coupled hydromagnetic wave excitation and ion acceleration upstream of the Earth's bow shock, $J$. Geophys. Res., 87, 5063, 1982.

Lepping, R. P., et al.. The WIND magnetic field investigation, Space Sci. Rev., 71, 207, 1995.

Lin, R. P., C.-I. Meng, and K. A. Anderson, 30- to 100$\mathrm{keV}$ protons upstream from the Earth's bow shock, $J$. Geophys. Res., 79, 489, 1974.

Lin. R. P., et al., A three-dimensional plasma and energetic particle investigation for the WIND spacecraft, Space $S c \imath$. Rev., 71, 125, 1995.

Luhmann, J. G., R. J. Walker, C. T. Russell, J. R. Spreiter, S. S. Stahara, and D. H. Williams, Mapping the magnetosheath field between the magnetopause and the bow shock: Implications for magnetospheric particle leakage, J. Geophys. Res., 89, 6829, 1984.

Luhmann, J. G., C. T. Russell, and R. C. Elphic, Spatial distributions of magnetic field fluctuations in the dayside magnetosheath, J. Geophys. Res., 91, 1711, 1986.

Mitchell, D. G.. and E. C. Roelof, Dependence of 50-keV upstream ion events at IMP $7 \& 8$ upon magnetic field bow shock geometry, J. Geophys. Res., 88, 5623, 1983.

Möbius, E., D. Hovestadt, B. Klecker, M. Scholer, F. M. Ipavich, C. W. Carlson, and R. P. Lin, A burst of energetic $\mathrm{O}^{+}$ions during an upstream particle event, Geophys. Res. Lett., 13, 1372, 1986.

Möbius, E., M. Scholer, N. Sckopke, H. Lühr, G. Paschmann, and D. Hovestadt, The distribution function of diffuse ions and the magnetic field power spectrum upstream of Earth`s bow shock, Geophys. Res. Lett., 14, 681, 1987.

Ogilvie, K. W., et al., SWE, A comprehensive plasma instrument for the WIND spacecraft, Space Sci. Rev., $\gamma_{1}$, $55,1995$.
Paschmann, G., N. Sckopke, S. J. Bame, J. R. Asbridge, J. T. Gosling, C. T. Russell, and E. W. Greenstadt. Association of low-frequency waves with suprathermal ions in the upstream solar wind, Geophys. Res. Lett., 6, 209, 1979.

Russell, C. T., R. C. Snare, J. D. Means, D. Pierce, D. Dearborn, M. Larson, G. Barr, and G. Le, The GGS/POLAR magnetic field investigation, Space Sci. Rev., 71, 563, 1995.

Russell, C. T., G. Le, P. Chi, X.-W. Zhou, J.-H. Shue, S. M. Petrinec, P. Song, F. R. Fenrich, and J. G. Luhmann, The extreme compression of the magnetosphere on May 4. 1998, as observed by the POLAR spacecraft, Adv. Space Res., in press, 1999.

Sarris, E. T., S. M. Krimigis, and T. P. Armstrong, Observations of magnetospheric bursts of high-energy protons and electrons at $\sim 35 R_{E}$ with $\operatorname{Imp} 7, J$. Geophys. Res., 81, 2341, 1976.

Scholer, M., F. M. Ipavich, G. Gloeckler, and D. Hovestadt, Conditions for acceleration of energetic ions $\geq 30 \mathrm{keV}$ associated with the Earth's bow shock, J. Geophys. Res., $85,4602,1980$.

Scholer, M., F. M. Ipavich, G. Gloeckler, D. Hovestadt, and B. Klecker, Leakage of magnetospheric ions into the magnetosheath along reconnected field lines at the dayside magnetopause, J. Geophys. Res., 86, 1299, 1981.

Scholer, M., E. Möbius, L. M. Kistler, B. Klecker, and F. M. Ipavich, Multispacecraft observations of energetic ions upstream and downstream of the bow shock, Geophys. Res. Lett., 16, 571, 1989.

Scudder, J., D. L. Lind, and K. W. Ogilvie, Electron observations in the solar wind and magnetosheath, J. Geophys. Res., 78, 6535, 1973.

Scudder, J. D., et al., Hydra - A 3-dimensional electron and ion hot plasma instrument for the POLAR spacecraft of the GGS mission, Space Sci. Rev., 71, 459, 1995.

Scudder, J. D., P. A. Puhl-Quinn, F. S. Mozer, K. W. Ogilvie, J. R. Wygant, and C. T. Russell, Generalized Walén tests for rotational discontinuities using electron flow velocities, J. Geophys. Res., $104,19.817,1999$ a.

Scudder, J. D., P. A. Puhl-Quinn, F. S. Mozer, K. W. Ogilvie, and C. T. Russell, The Generalized Walén tests throughout the May 4, 1998 magnetopause traverse, Eos Trans. $A G U, 80(17)$, Spring Meet. Suppl., S289, 1999b.

Sibeck, D. G., R. W. McEntire, A. T. Y. Lui, R. E. Lopez, A. M. Krimigis, R. B. Decker. L. J. Zanetti, and T. A. Potemra, Energetic magnetospheric ions at the dayside magnetopause: Leakage or merging?; J. Geophys. Res., 92, $12,097,1987$.

Speiser, T. W., D. J. Williams, and H. A. Garcia, Magnetospherically trapped ions as a source of magnetosheath energetic ions. J. Geophys. Res., 86, 723, 1981.

Spreiter, J. R., and S. S. Stahara, Magnetohydrodynamic and gasdynamic theories for planetary bow waves, in Collisionless Shocks in the Heliosphere: Reviews of Current Research, Geophys. Monogr. Ser., vol. 35, edited by B. T. Tsurutani and R. G. Stone, p. 85, AGU, Washington, D. C., 1985.

Trattner, K. J., E. Möbius, M. Scholer, B. Klecker, M. Hilchenbach, and H. Lühr, Statistical analysis of diffuse ion events upstream of the Earth's bow shock, J. Geophys. Res., 99, 13,389, 1994.

West, H. I., Jr., and R. M. Buck, Observations of $>100-\mathrm{keV}$ protons in the Earth's magnetosheath, J. Geophys. Res., $81,569,1976$.

Wilken, B., W. Weiss, D. Hall, M. Grande, F. Soraas, and J. F. Fennell, Magnetospheric ion composition spectrometer onboard the CRRES spacecraft, J. Spacecraft Rockets, 29, $585,1992$. 
Zwan, B. J., and R. A. Wolf, Depletion of solar wind plasma. near a planetary boundary, J. Geophys. Res., 81, 1636, 1976.

S.-W. Chang and J. D. Scudder, Department of Physics and Astronomy, The University of Iowa, Iowa City, IA 52242. (swc@space-theory.physics.uiowa.edu)

J. F. Fennell, The Aerospace Corporation, Mail Stop M2259, Los Angeles, CA 90009.

R. Friedel, Los Alamos National Laboratory, NIS-2, MS 436, Los Alamos, NM 87544.
S. A. Fuselier, W. K. Peterson, and K. J. Trattner, Lockheed Martin Advanced Technology Center, Dept. H1-11, Building 255, 3251 Hanover Street, Palo Alto, CA 94304.

R. P. Lepping, NASA Goddard Space Flight Center, Code 696.0, Greenbelt, MD 20771.

C. T. Russell, Institute of Geophysics and Planetary Physics, University of California, Los Angeles, CA 90095.

H. E. Spence, Boston University, Department of Astronomy and Space Physics, 725 Commonwealth Ave., Boston, MA 02215 .

(Received July 23, 1999; revised October 21, 1999; accepted October 22, 1999.) 